\title{
Dynamic mechanical characterization of two-photon-polymerized SZ2080 photoresist
}

\author{
L. Pertoldi, ${ }^{1, a)}$ V. Zega, ${ }^{2, \text { b) }}$ C. Comi, ${ }^{2, c)}$ and R. Osellame ${ }^{1,3, d)}$ \\ 1) Institute for Photonics and Nanotechnologies, CNR, 20133 Milano, Italy \\ ${ }^{2)}$ Department of Civil and Environmental Engineering, Politecnico di Milano, 20133 Milano, \\ Italy \\ 3) Department of Physics, Politecnico di Milano, 20133 Milano, Italy
}

(Dated: 6 October 2020)

Two-photon polymerization (2PP) is a material processing technique employed for the production of high-resolution micro-structures. The high potential of this technique in the fabrication of structured materials, or metamaterials, have recently attracted a significant research interest. To proceed towards real applications, the mechanical properties of the material obtained by $2 \mathrm{PP}$ should be known. These properties depend on all the process parameters, which affect the cross-linking between the polymeric chains, and very few results are available in the literature. In this work we perform a systematic characterization of the elastic properties of femtosecond-laser-polymerized SZ2080 (hybrid organic-inorganic photoresist) by combining dynamic experimental tests and numerical simulations on properly designed microstructures. Studying the resonance frequencies of micro-cantilevers, we demonstrate the possibility of tailoring the mechanical properties of the material by changing the laser irradiation conditions. This result paves the way to the use of 2PP for the fabrication of microdevices operating in a dynamic regime with optimized material properties.

\section{INTRODUCTION}

In recent years two-photon polymerization (2PP) emerged as one of the most important material processing techniques for the fabrication of mesoscale ${ }^{1}$ and sub-micrometric structures $^{2}$ with high spatial resolution. $2 \mathrm{PP}$ is an additive manufacturing technique, which combines the simplicity, versatility, and low cost of macroscopic three-dimensional (3D) printing techniques with the high resolution typical of conventional microfabrication techniques. The $2 \mathrm{PP}$ allows the single-step production of high-resolution 3D micro-structures with enormous advantages with respect to the usual multi-step lithographic techniques. Two-photon polymerization is achieved through the focusing of ultrashort infrared laser pulses inside a photoresist that absorbs linearly only the UV radiation and is normally transparent at the infrared frequencies. Polymerization with infrared light is possible only through a lowerefficiency nonlinear process, such as two-photon absorption. $2 \mathrm{PP}$ requires high peak intensity, for this reason femtosecond laser sources and high numerical aperture objectives are used. The polymerization occurs only in the ellipsoid-shaped region, called voxel, around the focal volume, where the laser intensity is above the threshold. By properly moving the sample with respect to the laser beam it is possible to obtain 3D geometries. Exploiting the nonlinearity of the absorption process and setting the intensity just above threshold, it is possible to overcome the diffraction limit, obtaining resolutions down to tens of nanometres using a laser beam with wavelength of several hundreds of nanometers ${ }^{3-6}$. For its unique capability to produce 3D microstructures with sub-diffraction

a)Electronic mail: luca.pertoldi@ polimi.it.

b)Electronic mail: valentina.zega@polimi.it.

c)Electronic mail: claudia.comi@polimi.it.

d)Electronic mail: roberto.osellame@ polimi.it. limit resolution, 2PP has many possible applications, such as biomedics $^{7-15}$, micro-optics ${ }^{16-18}$, micro-fluidics ${ }^{19-21}$, micromechanics $^{22,23}$ and photonics ${ }^{24,25}$.

To optimize the micro-structures for many of the abovelisted applications, the mechanical properties of the polymerized material should be known and possibly adjustable. The latter, however, are hardly known as they depend on many process parameters such as the laser power, the writing speed and the writing density. In the literature there are few studies focused on the evaluation of mechanical properties of photopolymerized resins for $2 \mathrm{PP}^{26}$. In Lemma et al. $^{27}$, the elastic properties of several polymers cross-linked by two-photon lithography at varying laser power were estimated through static and dynamic tests on microbeams. Raman spectroscopy and nanoindentation tests were instead exploited to characterize the IP-L 780 resin $^{28}$, while nanoindentation on microbeams was used to probe different acrylatebased materials ${ }^{29}$. A recent paper ${ }^{30}$ studied the influence of key process parameters, including the writing speed and density, on the Young's modulus of two-photon-polymerized IPDip resin by means of tension and compression tests on microstructures, combined with Raman spectroscopy.

In the present work, we focus on a different resin, the widely-used, hybrid organic/inorganic, negative photoresist SZ2080. SZ2080 has many advantages, such as biocompatibility, long-term stability, chemical and electrochemical inertia, good optical transmission, low distortion and negligible shrinkage after solvent washing with no need for shape compensation in the irradiation step ${ }^{31}$. Previous studies reported a value of the Young's modulus of UV-polymerized SZ2080 obtained by nanoindentation ${ }^{32}$, which is about ten times greater than the one indirectly measured through a quite complex experimental setup on the same photoresist cross-linked by $2 \mathrm{PP}^{33}$. Another recent work ${ }^{12}$ performed a static characterization of the mechanical properties of SZ2080 by microindentation and nanoindentation. However, a systematic inves- 
tigation of the influence of the fabrication parameters on the dynamical behaviour of this material is not available so far.

The purpose of this work is the experimental mechanical characterization of the two-photon polymerized SZ2080 at room temperature. The focus is on the effect of the different writing parameters: power, writing speed, distance between lines in $x y$-plane and distance between planes along the $z$-direction, on the resulting material elastic properties. The elastic moduli are obtained from resonance frequency measurements of micro-structures fabricated through 2PP and actuated by a piezoelectric disc. Two different micro-beams are designed to allow for the dynamic measurement of the longitudinal and shear moduli. In addition, the effect of the direction of writing and the related anisotropic effects are investigated. This study provides a database to systematically tailor process parameters depending on the required mechanical properties for SZ2080 photoresist.

\section{MATERIALS AND METHODS}

\section{A. Materials}

In the literature, several functional resins and photoresists for $2 \mathrm{PP}$ have been used ${ }^{34}$, which provide polymerized structures that differ in terms of ultimate resolution and mechanical properties. Photoinitiators also play a role in the $2 \mathrm{PP}$ process $^{35}$, thus underlining the need of a proper choice of materials to achieve the desired properties.

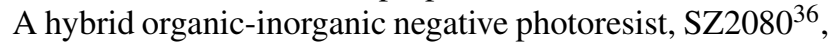
is used in this work. SZ2080 is made of two components: methacryloxypropil trimethoxysylane (MAPTMS, $\geq$ 97\%, Sigma-Aldrich) and zirconium propoxide (ZPO, 70\% in propanol, Sigma-Aldrich), where the addition of ZPO enhances the material's mechanical stability. The photoresist is also doped with 1\% wt Irgacure 369 (IRG, Sigma-Aldrich) as photoinitiator.

A 50:50 solution in volume of 4-methyl-2-pentanone $(\geq$ 99.5\%, Sigma-Aldrich) and isopropyl alcohol ( $\geq 99.8 \%$, Sigma-Aldrich) was used as solvent to remove the unpolymerized resist from the irradiated structures ${ }^{37}$.

The mass density of this material has been measured on a macroscale prototype, polymerized through UV light, and is equal to $\rho=1200 \mathrm{Kg} / \mathrm{m}^{3}$ in good agreement with results already available in the literature ${ }^{11}$.

\section{B. Direct writing procedure}

A scheme of the experimental set-up for the fabrication of $3 \mathrm{D}$ microstructures by $2 \mathrm{PP}$ is shown in Fig. 1. The femtosecond laser source (Topica, FemtoFiber pro NIR) operates at its second harmonic emitting mode-locked pulses at $780 \mathrm{~nm}$ wavelength. This source has a repetition rate of $80 \mathrm{MHz}$ and a pulse length of about $80 \mathrm{fs}$. The photopolymerized structure is generated in a layer-by-layer format. Each layer is formed using $x-y$ linear stages (ANT95-50-XY, Aerotech) to move the structure with respect to the laser beam. Movement

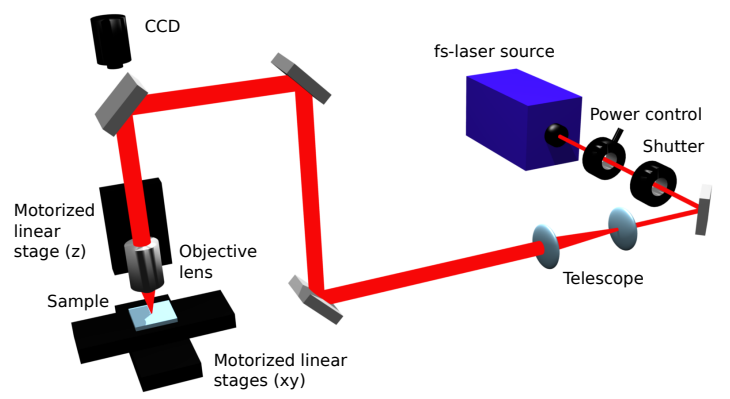

FIG. 1. General scheme of the fabrication setup used in this work.

along the $z$-axis is achieved using a high resolution linear stage (ANT130-035-L-ZS, Aerotech) that translates the objective lens. To achieve the tight focusing conditions required for 2PP, we used an oil-immersion objective (Carl Zeiss, PlanApochromat, 100x n and 1.4 NA). Sample irradiation is controlled by a fast galvanometric shutter, while beam intensity is regulated by a combination of a polarizer and a waveplate. For the real-time monitoring of the photopolymerization process, a CCD camera is mounted behind a dichroic mirror. Taking advantage of the fact that the refractive index of SZ2080 changes during polymerization, the illuminated structures become visible during the irradiation process. The resin is dropcasted on a glass coverslip and mounted upside-down in the fabrication set-up. To avoid the distortion of the focused laser beam by the already-built structures, they are fabricated layerby-layer from the deepest point in the resin to the glass coverslip.

To fabricate a sample or a particular micro-structure the three-dimensional image is first converted to stereolithographic (STL) file. The final STL file is input to the fabrication program that slices the 3D model into different planes at a fixed distance $d_{z}$ along the $z$ axis and scans the area of every plane by writing lines along the $x$ - and/or the $y$-axis at distances $d_{x}$ and $d_{y}$, see Fig. 2. The structure is hence fabricated plane-by-plane and line-by-line until the whole structure is built ${ }^{38}$.

After the photopolymerization process, in order to remove the unpolymerized resin, the samples are developed in a suitable solvent for 20 minutes.

\section{Micro-beams for mechanical testing}

To achieve a faithful and reliable characterization, mechanical tests are carried out on specimens fabricated at the same scale and with the same process of a typical micro-structure made by 2PP in SZ2080. Furthermore, to identify the elastic material behavior, at least two independent parameters (i.e. the Young's modulus $E$ and the shear modulus $G$ ) must be measured. To that purpose we designed two sets of microbeams with dimensions compatible with the fabrication system described in Section II B. The first set of structures is 


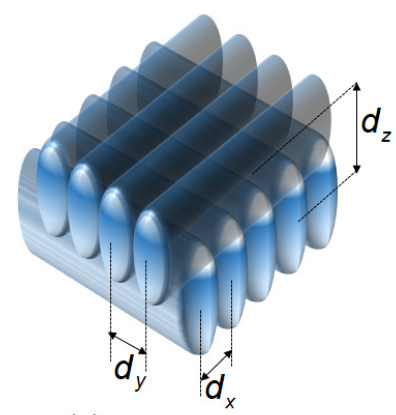

(a)

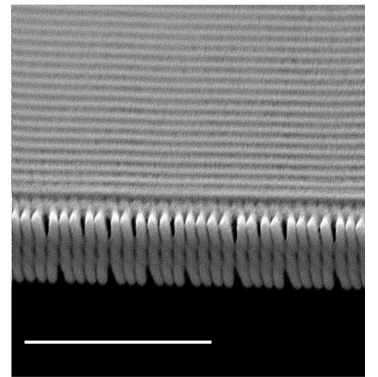

(b)
FIG. 2. Multi-voxel-lines texture: (a) scheme and writing distance definition (b) SEM image. The scale bar is equivalent to $5 \mu \mathrm{m}$ in the SEM image.

made by slender cantilever beams designed to oscillate in bending with a first flexural mode at a frequency in the order of some tens of $\mathrm{kHz}$, while the second one is made by cantilever beams with and eccentric mass at the tip to promote torsional oscillations. The measure of the flexural and torsional natural frequencies of these two sets of structures, allows the indirect determination of the elastic moduli.

To investigate the influence of the fabrication process on such moduli, many cantilevers have been fabricated with different writing parameters. In particular, scans of power, writing speed, distance between the lines in the $x y$-plane and distance between planes along the $z$-direction have been performed and studied.

In addition, since the fabrication process may result in a non-isotropic material, the same structures have been fabricated in three ways: with all the lines of each plane along the longitudinal direction (direction of the axis of the cantilever), with all the lines of each plane along the transverse direction and with each plane made of lines in the perpendicular direction with respect to the previous plane as in Fig. 2.

\section{Oscillation measurements}

The natural frequencies of the fabricated cantilevers have been measured through the experimental setup shown in Fig. 3.

The sample is mechanically fixed on a piezoelectric disc, a PRYY+0354 by PICeramics, that is driven at the desired frequency by the arbitrary function generator (Tektronix, AFG 3011C). A standard He-Ne laser with a wavelength of $632.8 \mathrm{~nm}$ (Melles Griot, 05-lhp-151) is focused by an objective (Olympus, MPlanApo N, 100x and 0.95NA) on the tip of the cantilever. The reflected beam, after passing through an iris, is detected by a silicon photodiode (Thorlabs, PDA36AEC). When the cantilever oscillates, the reflection at the tip is periodically deflected out of the iris aperture, thus causing a modulation of the transmitted power. The oscillating signal coming from the photodiode is then sent to a digital oscilloscope (Tektronix, DPO 2024B). A sweep on the forcing frequency is performed and the natural frequency of the pro-

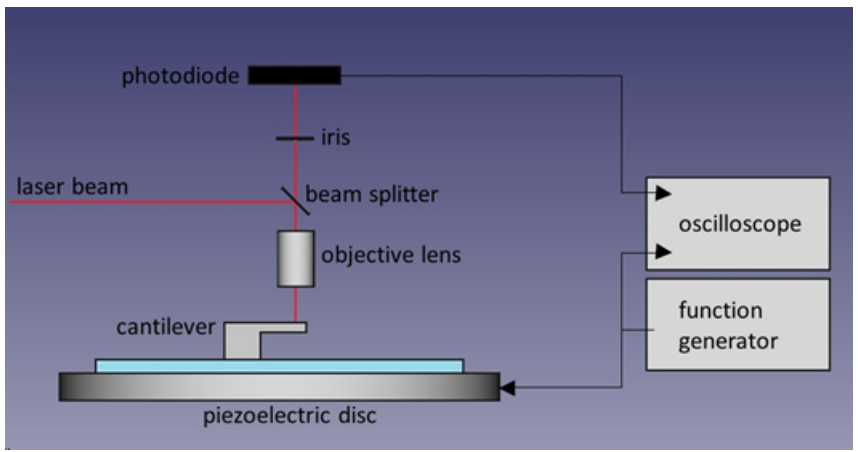

FIG. 3. Not-to-scale scheme of the characterization setup: the red line is the laser beam path while the black arrows represent the flux of electrical signal.

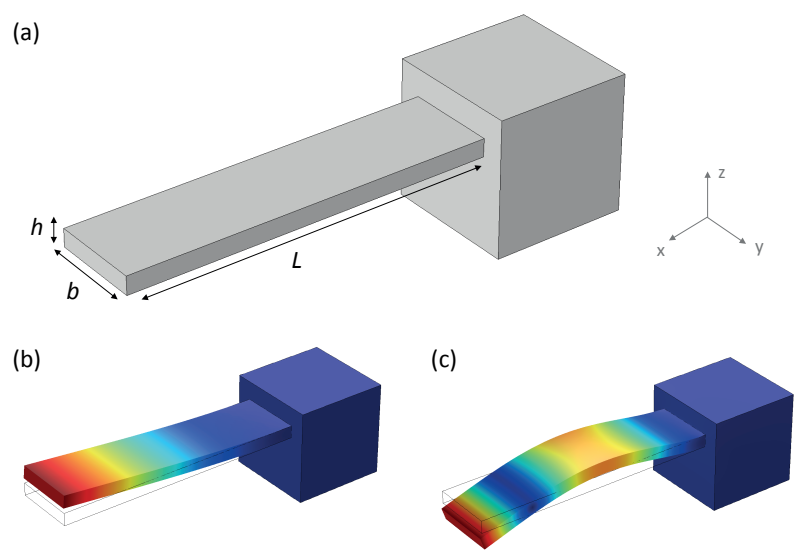

FIG. 4. (a) Cantilever beam with nominal dimensions $L=80 \mu \mathrm{m}$, $b=20 \mu \mathrm{m}$ and $h=4 \mu \mathrm{m}$. (b) First and (c) second vibration modes. The contour of the displacement amplitude is shown in color.

totype under test is identified with the forcing frequency that causes the maximum response amplitude.

\section{MODELING AND SIMULATION}

\section{A. Bending structures}

The bending structures are cantilever beams attached to the substrate by a bulky block of dimensions $30 \mu \mathrm{m} \times 30 \mu \mathrm{m} \times$ $30 \mu \mathrm{m}$, as shown in Fig. 4a, where the nominal dimensions are indicated.

For slender beams of length $L$ and rectangular cross section of height $h$, the bending frequencies, estimated by the linear beam theory, read:

$$
f=\frac{\alpha}{2 \pi L^{2}} \sqrt{\frac{h^{2} E}{12 \rho}}
$$

where $\rho$ is the mass density, $E$ the Young's modulus and $\alpha=$ 3.515 for the first mode and $\alpha=22.034$ for the second one. Hence, in the approximation of the Bernoulli beam theory, the 
TABLE I. Nominal frequencies of the clamped beam in bending.

\begin{tabular}{lcc}
\hline \hline & Beam theory & FE simulation \\
\hline$f_{1}$ & $92139 \mathrm{~Hz}$ & $90936 \mathrm{~Hz}$ \\
$f_{2}$ & $577580 \mathrm{~Hz}$ & $561910 \mathrm{~Hz}$ \\
\hline \hline
\end{tabular}

frequency is independent from the beam width $b$ and from the material Poisson's coefficient $v$; if the material density is known, the measurement of the frequency allows to compute the Young's modulus $E$.

A more precise estimation of the frequencies, which accounts for the deviation from the ideal Bernoulli theory and for the presence of the block instead of a perfect clamping constraint, is obtained numerically through a finite element (FE) simulation in COMSOLMultiphysics ${ }^{\circledR}$. The geometry of the cantilever is discretized with a regular mesh made by around 5000 quadratic volume elements to guarantee at least three finite elements in the thickness and a consequent high accuracy of the numerical solution. Modal analyses are then performed to simulate the natural frequencies of the two bending modes of the cantilevers shown in Figs. 4b-c.

Fixing the geometric dimensions as in Fig. 4a and $E=$ $1 \mathrm{GPa}$, the analytic (Eq. 1) and numeric frequencies are computed as reported in Table I. One can observe a difference of about $1.3 \%$ and $2.8 \%$ for the first and second modes, respectively mainly due to the block compliance. However, the analytic formula can be used in the design phase and highlights the dependence of the frequency on the square root of $E$, which will be exploited in the following to identify the Young's modulus from the experimental data. Note that even though not very influential on the bending frequency, a value of the Poisson's ratio is required for the numerical analyses; a value $v=0.4$ is considered, which is in the range reported in the literature ${ }^{27,39}$ for similar materials.

\section{B. Torsional structures}

The torsional structures are cantilever beams, with a tip eccentric mass $M$ designed to promote the torsional oscillation, see Fig. 5. In this case, the presence of the anchoring block and the asymmetric position of the tip mass with respect to the torsional axis causes a coupling between the torsional and flexural behaviours of the beam.

The simple analytic formula ${ }^{40}$ for the torsional frequency is hence not valid and a numerical approach is followed. Both the flexural mode of the structure shown in Fig. 5b and the torsional mode shown in Fig. 5c are excited, the corresponding frequencies are measured and the Young's and shear moduli are identified through numerical simulations in COMSOLMultiphysics $^{\complement}$, this time with a regular mesh made by around 7000 quadratic volume elements.

With the nominal dimensions given in Fig. $5 \mathrm{a}(L=65 \mu \mathrm{m})$ and assuming $G=0.36 \mathrm{GPa}$ (i.e. $E=1 \mathrm{GPa}$ and $v=0.4$ ), the numerically computed flexural frequency is $82804 \mathrm{~Hz}$, while the torsional counterpart is $411690 \mathrm{~Hz}$. (a)
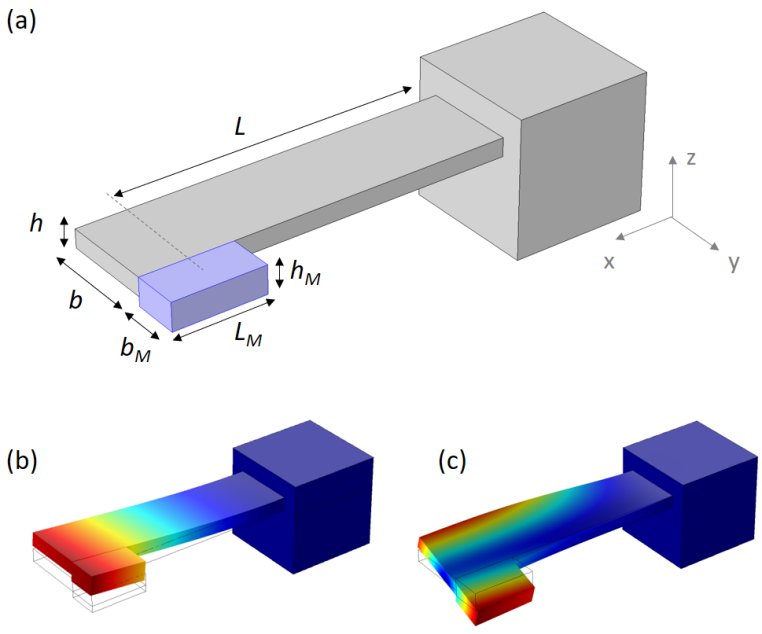

FIG. 5. (a) Torsional structure with nominal dimensions $L=65 \mu \mathrm{m}$ or $L=45 \mu \mathrm{m}, b=20 \mu \mathrm{m}, h=4 \mu \mathrm{m}, L_{M}=20 \mu \mathrm{m}, b_{M}=10 \mu \mathrm{m}$ and $h_{M}=6 \mu \mathrm{m}$. (b) Flexural and (c) torsional modes. The contour of the displacement amplitude is shown in color.
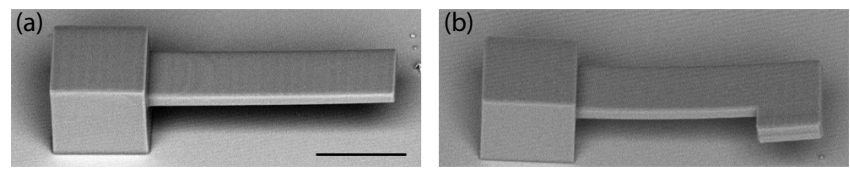

FIG. 6. SEM image of the fabricated (a) flexural and (b) torsional structures. The scale bar is equivalent to $30 \mu \mathrm{m}$ for both panels.

\section{Elastic properties}

After fabrication, the real dimensions of each cantilever have been measured with table-top scanning electron microscope (SEM) - Phenom World, Phenom Pro. SEM images of the cantilevers are reported in Fig. 6.

The measured dimensions are inserted in a 3D FE model used to compute, through modal analyses, the first two bending frequencies of the cantilevers. In view of the linearity assumed for the material, as the bending frequency is proportional to the square root of the Young's modulus $E$, the analyses are performed with a unit value of $E$, and the unknown Young's modulus is obtained from the ratio between the measured $f_{\exp }$ and the numerical frequencies $f_{E=1}$ as

$$
E=\left(\frac{f_{\exp }}{f_{E=1}}\right)^{2}
$$

Also the second bending frequency is exploited similarly to check the soundness of the procedure. The cantilever beams with an eccentric mass, designed to oscillate in torsion are also measured and simulated through modal analyses in COMSOLMultiphysics ${ }^{\circledR}$. 
TABLE II. Writing process parameters: reference value and considered range of variation.

\begin{tabular}{lccc}
\hline \hline Parameter & Reference & $\min$ & $\max$ \\
\hline Power $[\mathrm{mW}]$ & 20 & 15 & 25 \\
Writing speed $[\mu \mathrm{m} / \mathrm{s}]$ & 1000 & 500 & 3500 \\
$d_{x}[\mu \mathrm{m}]$ & 0.3 & 0.02 & 0.5 \\
$d_{z}[\mu \mathrm{m}]$ & 0.5 & 0.1 & 0.6 \\
\hline \hline
\end{tabular}

\section{RESULTS AND DISCUSSION}

Starting from a reference set of writing parameters, namely: laser power $P=20 \mathrm{~mW}$, writing speed $v=1000 \mu \mathrm{m} / \mathrm{s}$, distance between lines $d_{x}=d_{y}=0.3 \mu \mathrm{m}$ and $d_{z}=0.5 \mu \mathrm{m}$, several sets of bending and torsional beams have been fabricated by changing one single writing parameter at a time. The interval of variation for each parameter is specified in Table II.

Note that the number of prototypes fabricated for each combination of writing parameters is different. 136 cantilever beams have been fabricated, out of which 28 with the reference parameters reported in Table II and 108 with other combinations of parameters. Mean values and standard deviations are then computed on sets of data of different numerosity. When only one prototype is available, the standard deviation is not reported.

\section{A. Young's modulus}

Flexural structures as the one shown in Fig. 4 are tested to identify the Young's modulus from their natural frequencies through Eq. (2). The first and second flexural modes are excited and the natural frequencies are measured through an oscilloscope as described in Section II B. The first natural frequencies of the different prototypes are employed for the identification of the Young's modulus, while the second natural frequencies are considered for the validation of the obtained results. Figure 7 shows the output signals measured on a cantilever fabricated with the reference writing parameters reported in Table II and excited at its first and second natural frequencies, $96900 \mathrm{~Hz}$ and $597100 \mathrm{~Hz}$, respectively. It is worth noting that the accuracy we achieved in the measurement of the first natural frequency is much higher than the one of the second frequency because a much smaller signal is measured when the second flexural mode of the cantilevers is excited, thus causing uncertainties in the frequency identification.

Considering an initial set of 7 cantilevers, all fabricated with the reference writing parameters, the Young's modulus identified by the first frequency is $992 \pm 14 \mathrm{MPa}$, while using the second frequency one obtains $996 \pm 16 \mathrm{MPa}$. The two values are in very good agreement (well within the standard deviations) and are a clear proof of the consistent modelling of the cantilever dynamical behaviour.

In the following, fortified by the validation performed through the measurement of the second flexural frequency of the fabricated prototypes, we focus on the first flexural mode (a)
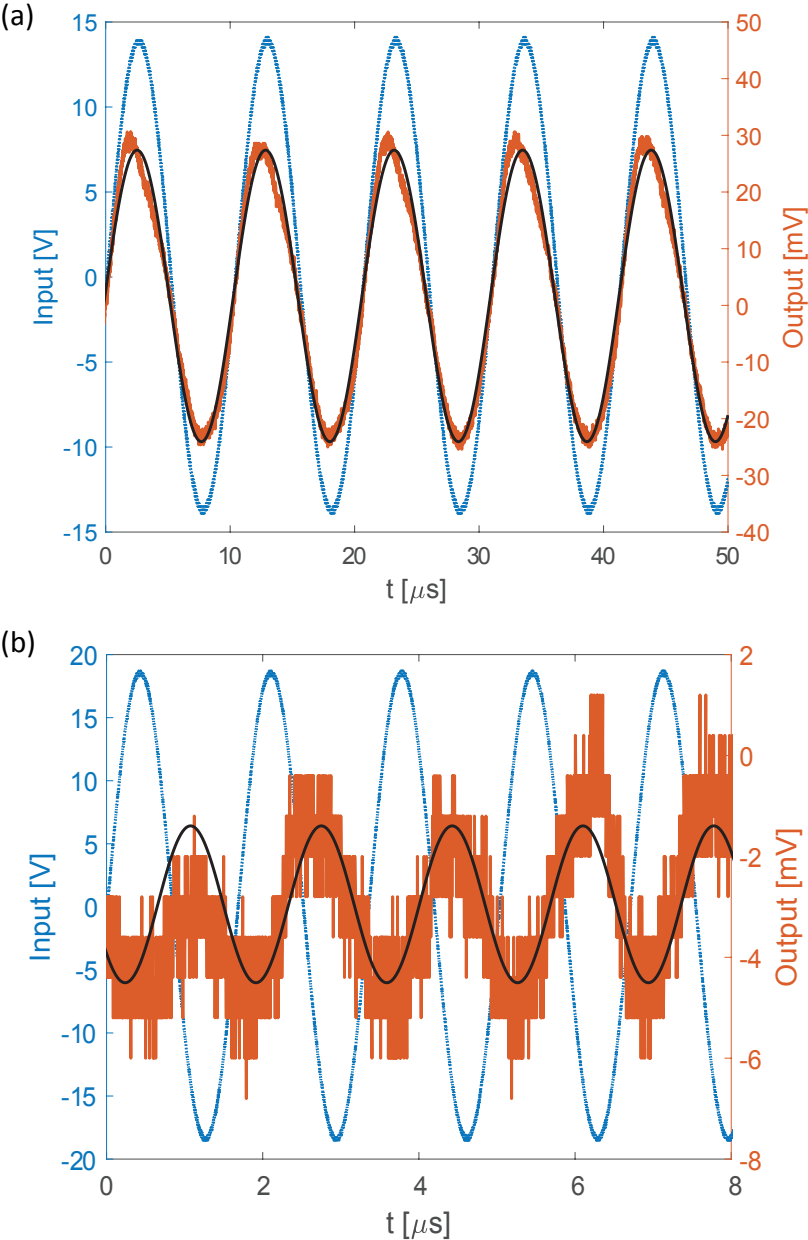

FIG. 7. Input (blue dashed) and output (orange) signals measured on the flexural structures when the (a) first and the (b) second flexural modes are excited. The black line is a fit to the data.

of the whole set of 28 cantilevers fabricated with the reference parameters. The Young's modulus identified from the first mode turns out to be $992 \pm 19 \mathrm{MPa}$.

The dependence of the Young's modulus on the distance $d_{x}=d_{y}$ is studied in Fig. 8. A non-monotonic behaviour is found with a maximal stiffness $(E=1076 \pm 34 \mathrm{MPa}$ on 7 specimens) achieved for a distance of about $0.1 \mu \mathrm{m}$. The trend can be explained by the fact that at this power level, the in-plane voxel radius is about $0.3 \mu \mathrm{m}$ hence very small distances $d_{x}$ lead to a large irradiation overlap that can damage and disrupt the structure, thus causing a significant decrease of the Young's modulus ( $43 \%$ reduction for $d_{x}=0.02 \mu \mathrm{m}$ ). On the other side, a large distance $d_{x}$ leads to a more compliant material because of a lower irradiation overlap leading to a weakly-polymerized material ( $15 \%$ reduction for $d_{x}=0.5$ $\mu \mathrm{m})$.

In Fig. 9 the variation of the Young's modulus with the distance $d_{z}$ is reported. Also in this case the trend is nonmonotonic and shows a maximum for $d_{z}=0.5 \mu \mathrm{m}$. The maximum is obtained for a bigger distance with respect to the re- 


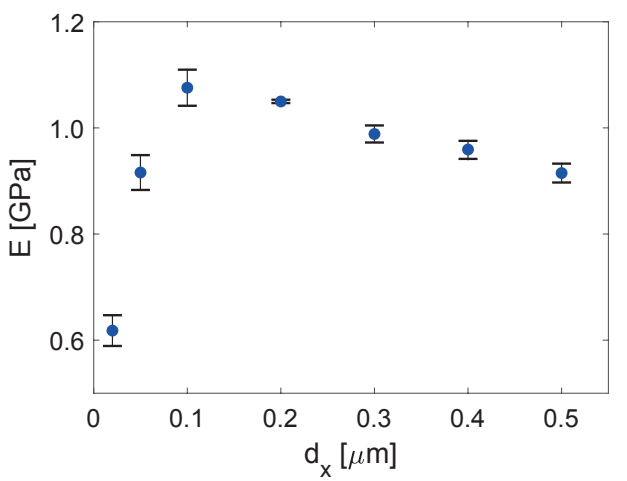

FIG. 8. Young's modulus vs distance $d_{x}=d_{y}$; laser power $20 \mathrm{~mW}$ writing speed $v=1000 \mu \mathrm{m} / \mathrm{s}$ and $d_{z}=0.5 \mu \mathrm{m}$.

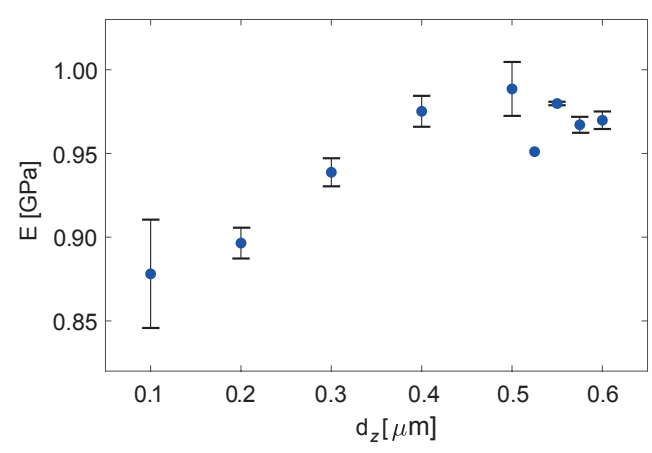

FIG. 9. Young's modulus vs distance $d_{z}$; laser power $20 \mathrm{~mW}$ writing speed $v=1000 \mu \mathrm{m} / \mathrm{s}$ and $d_{x}=d_{y}=0.3 \mu \mathrm{m}$.

sults reported in Fig. 8 because of the ellipsoidal shape of the voxel with a vertical axis of about $1 \mu \mathrm{m}$ (Fig. 2).

Figure 10 shows the variation of the Young's modulus with the laser power: increasing the laser power from $15 \mathrm{~mW}$ to $25 \mathrm{~mW}$, the Young's modulus decreases by following a quadratic trend, which suggests the presence of a peak value around $15 \mathrm{~mW}$.

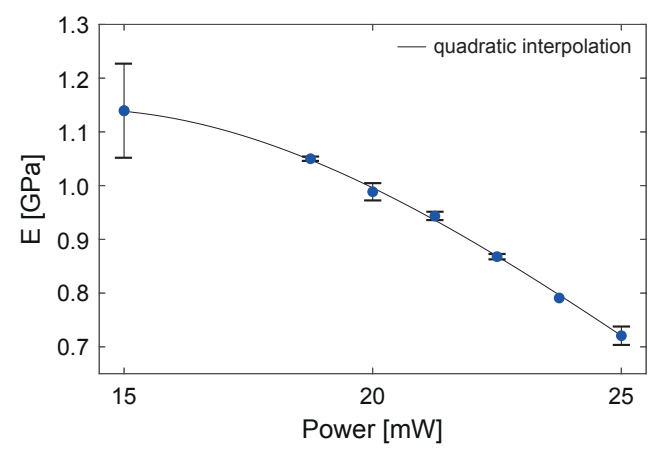

FIG. 10. Young's modulus vs laser power; writing speed $v=1000 \mu \mathrm{m} / \mathrm{s}$ and distance between lines $d_{x}=d_{y}=0.3 \mu \mathrm{m}$ and $d_{z}=0.5 \mu \mathrm{m}$.

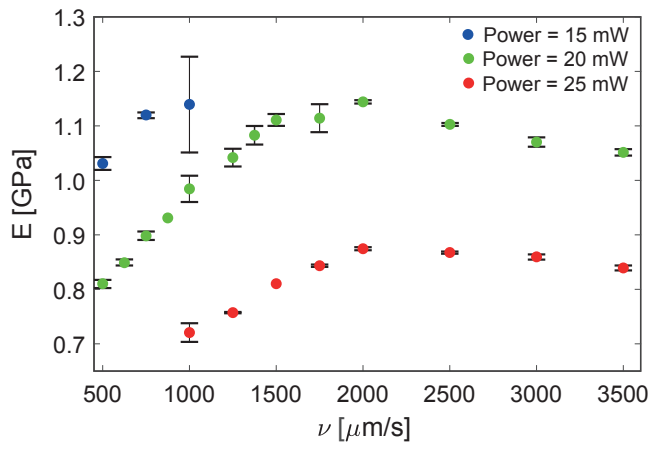

FIG. 11. Young's modulus vs writing speed; laser power $20 \mathrm{~mW}$ and distance between lines $d_{x}=d_{y}=0.3 \mu \mathrm{m}$ and $d_{z}=0.5 \mu \mathrm{m}$.

This behaviour is better understood if analysing the combined dependence of the elastic modulus on irradiation power and speed, as reported in Fig. 11. While it is true that at the reference speed of $1000 \mu \mathrm{m} / \mathrm{s}$ the Young's modulus E is higher at $15 \mathrm{~mW}$ irradiation than at $20 \mathrm{~mW}$, one can note that for a larger speed of about $2000 \mu \mathrm{m} / \mathrm{s}$ the same peak value of $\mathrm{E}$ is achieved also for $20 \mathrm{~mW}$ power irradiation. This seems to indicate that, more than an optimum for power and speed separately, there is an optimal value of energy deposited per unit length that depends on the ratio Power $/ v$. It is interesting to observe that for the highest irradiation power of $25 \mathrm{~mW}$, the same peak value of $\mathrm{E}$ is not achieved for any speed, which means that the pulse energy associated with this irradiation power is already above the damage threshold irrespectively of the writing speed. On the other side, we could expect to achieve the same maximum stiffness for the material polymerized with lower power and lower speed (keeping the deposited energy per unit length almost constant), however that region is not interesting because lower irradiation speed means increasing significantly the processing time to fabricate complex microstructures. As an example, a cantilever fabricated with the reference parameters requires an irradiation time of 4'13", while if we reduce the speed by a factor of 2 the irradiation time becomes 7'56". In order to avoid affecting the processing time, we believe that the best strategy is fixing the combination power-speed that provides the highest $\mathrm{E}$ with the largest speed, and, if needed, modulating the elastic response with the line distances or the power, but not the speed.

From all the above results one can conclude that the polymer SZ2080 obtained with 2PP has a Young's modulus that strongly depends on the fabrication parameters and that can be tailored in the range ( $0.6-1.1 \mathrm{GPa})$ by a suitable choice of the parameters combination.

\section{B. Shear modulus}

Torsional structures as the one shown in Fig. 5 are tested to identify the shear modulus. Flexural and torsional modes are excited to allow the identification of the Young's modulus and Poisson's ratio through the comparison between experiments 
and numerical predictions. Assuming isotropic behavior the shear modulus reads:

$$
G=\frac{E}{2(1+v)}
$$

As anticipated in Section III B, due to the intrinsic coupling between the flexural and torsional behaviours of these structures, it is not possible to identify the Young's modulus and the Poisson's ratio independently. An iterative procedure that allows the identification of the two materials parameters starting from the measured natural frequencies of the torsional structures is then carried out. The iterative procedure consists in hypothesizing the Young's modulus and the Poisson's ratio, computing the natural frequencies of the bending and torsional modes of the cantilevers with tip mass through modal analyses in COMSOLMultiphysics ${ }^{\circledR}$, correcting the estimations until the error between the experimental frequencies and the numerical ones computed with the identified material parameters is below $6 \%$.

Four torsional structures of length $L=65 \mu \mathrm{m}$ and two of length $L=45 \mu \mathrm{m}$ are tested through the experimental setup described in Section II D. The identified Young's modulus is $1.005 \mathrm{GPa} \pm 13 \mathrm{MPa}$ in good agreement with the results obtained from the flexural structures in the previous sections, while the identified Poisson's ratio is $0.42 \pm 0.03$, a bit higher, but close to the value of 0.4 , taken from the literature and used in this paper as a reference. Using Eq. (3), the values of the shear modulus $G=353.34 \pm 3.3 \mathrm{MPa}$ is obtained.

The larger uncertainty in the Poisson's ratio can be justified by noting that the natural frequencies of the measured torsional modes are much higher (i.e. $420-580 \mathrm{kHz}$ ) than their flexural counterparts (i.e. $80-170 \mathrm{kHz}$ ). As already observed in Section IV A, the output signal is lower and hence the measure less reliable in the high frequency range.

\section{Non-isotropic behavior}

The material obtained through $2 \mathrm{PP}$ is in general nonisotropic. The frequency measurements of cantilevers fabricated with different directions of writing allow to investigate this aspect. Instead of the usual cross ply scheme shown in Fig. 2, 10 cantilevers have been fabricated writing only in the longitudinal direction $x$ and 10 in the transverse direction $y$. All writing parameters are kept to the reference values of Table II.

Since the frequency of the cantilever depends on the stiffness in the longitudinal direction, from the first set of ten cantilevers, fabricated with a longitudinal writing, one can measure the stiffness in the writing direction $E_{x}$. For the same reason, from the second set of ten cantilevers, fabricated with a transverse writing, the Young's modulus in the direction normal to the writing direction, $E_{y}$, is identified. The mean values thus obtained are $E_{x}=974 \pm 13 \mathrm{MPa}$ and $E_{y}=885 \pm 20 \mathrm{MPa}$. As expected, in the writing direction the stiffness is higher than in the transverse direction. Furthermore, one can note that the cross ply result, presented in Section IV A, $E=992$ $\pm 19 \mathrm{MPa}$, is only slightly higher than that achieved with longitudinal writing alone.

\section{Discussion}

Table III collects experimental values, available in the literature, of the elastic parameters $E$ and $v$ of several resins used for $2 \mathrm{PP}$. The last row refers to the present study. The whole set of data testifies the possibility to obtain, through $2 \mathrm{PP}$, microstructures with different stiffness, by changing the photoresist and/or the fabrication parameters, such as the laser power, the writing rate, and the interline distance. The large spread of results for the same material can be partially attributed to the different fabrication and measurement conditions.

In particular, we focus the discussion on the last four rows of Table III, which are all related to the SZ2080 resin employed in this work. The available data on the Young's modulus span from $0.14 \mathrm{GPa}^{33}$ to $2.8 \mathrm{GPa}^{12}$. The lowest reported values of the Young modulus ${ }^{33}$ are obtained by a complex indirect method that combines a glass cantilever and a polymer beam, while higher values are retrieved with nanoindentation measurements ${ }^{12,32}$. The Young's modulus obtained in this work sits in an intermediate range. We note that Skarmoutsou et al. ${ }^{32}$ characterize the mechanical properties on a UV-polymerized material, while Tickunas et al. ${ }^{33}$ perform the 2PP process at a laser wavelength of $1030 \mathrm{~nm}$ and a pulse repetition rate of $610 \mathrm{kHz}$, which is in turn different from the 780 - $800 \mathrm{~nm}$ wavelength and $80 \mathrm{MHz}$ combination, used in Flamourakis et al. ${ }^{12}$ and this work. A further difference regards the frequency range at which the characterization is performed: Tickunas et al. ${ }^{33}$ and Skarmoutsou et al. ${ }^{32}$ perform a static characterization, Flamourakis et al. ${ }^{12}$ implement a dynamic nanoindentation technique in the frequency range $0.1 \mathrm{~Hz}-$ $300 \mathrm{~Hz}$, while our work explores a higher frequency characterization regime at $100-500 \mathrm{kHz}$. All these aspects could partially explain the differences in the observed mechanical properties.

Finally, it is worth mentioning that in the present work, the Poisson's ratio is measured to fully characterize the mechanical properties of the polymer. The direct measure of such parameter is not common in the literature as reported in Table III.

\section{v. CONCLUSIONS}

The material properties of the two-photon-polymerized SZ2080 are characterized through a widespread experimental campaign on cantilever beams fabricated by spanning different combinations of writing parameters.

The results show that there is an optimal combination of values of power, writing speed and line distance that leads to the maximum Young modulus of about 1.1 GPa. However, by changing the fabrication parameters it is possible to tune the stiffness of the material with a significant reduction (up to $40 \%$ ) of the Young's modulus. In addition, we demonstrated a clear dependence of the Young's modulus on the 


\begin{tabular}{|c|c|c|c|c|}
\hline Paper & Resin & Test & $E[\mathrm{GPa}]$ & $v[-]$ \\
\hline Lemma et al. ${ }^{27}$ & IP-G 780 & micro-bending tests & $2.1-3.7$ & $0.45-0.5^{*}$ \\
\hline Lemma et al. ${ }^{27}$ & SU8 2100 & bending tests & 2.4- 3.0 & $0.45-0.5^{*}$ \\
\hline Lemma et al. ${ }^{27}$ & Ormocomp & bending tests & $0.8-1.2$ & $0.45-0.5^{*}$ \\
\hline Lemma et al. ${ }^{27}$ & IP-L 780 & micro-bending tests & $1.3-2.1$ & $0.45-0.5^{*}$ \\
\hline Jang et al. ${ }^{28}$ & IP-L 780 & nanoindentation and Raman spettroscopy & $0.57-0.7$ & N.A. \\
\hline Cicha et al. ${ }^{29}$ & ETA, Sartomer 415 & nanoindentation on micro-cantilever & $1.2-1.65$ & N.A. \\
\hline Cicha et al. ${ }^{29}$ & TTA, Genomer 1330 & nanoindentation on micro-cantilever & $1.4-2.65$ & N.A. \\
\hline Lemma et al. ${ }^{27}$ & IP-Dip & micro-bending tests & $0.75-3.6$ & $0.45-0.5^{*}$ \\
\hline Bauer et al. ${ }^{30}$ & IP-Dip & uniaxial compression and tension experiments & $0.6-3.6$ & N.A. \\
\hline Bayindir et al. ${ }^{41}$ & SR499/368 & atomic force microscopy/nanoindentation & $0.44 \pm 0.03 / 0.52 \pm 0.11$ & N.A. \\
\hline Tickunas et al. ${ }^{33}$ & SZ2080 & indirect method & $0.138-0.188$ & N.A. \\
\hline Skarmoutsou et al. ${ }^{32}$ & $\mathrm{SZ} 2080 \dagger$ & nanoindentation & $1.5-1.8$ & N.A. \\
\hline Flamourakis et al. ${ }^{12}$ & SZ2080 & dynamic indentation & $1.4-2.8$ & $0.49 \pm 0.002$ \\
\hline This work & SZ2080 & bending and torsion vibration of cantilevers & $0.6-1.1$ & $0.42 \pm 0.03$ \\
\hline
\end{tabular}

TABLE III. Mechanical properties of different 2PP resins. ${ }^{*}$ Poisson's values reported in Lemma et al. ${ }^{27}$ are typical values for rubber like materials and are not directly measured. † Although the main components, i.e. MAPTMS and ZPO, are the same, the material used in this work differs from standard SZ2080 for the use of 2-(dimethylamino)ethyl methacrylate (DMAEMA) instead of methacrylic acid (MAA).

writing direction, enabling the straightforward realization of anisotropic micromechanical structures by two-photon polymerization. The Poisson's ratio is also experimentally characterized through the proper design of cantilevers with a tip mass.

The results of this work provide reliable data for future modelling of 3D microstructures fabricated by $2 \mathrm{PP}$ in SZ2080. In addition, they prove the possibility of a significant tailoring of the mechanical properties of these structures by a proper choice of the fabrication parameters. This paves the way to the realization of optimized structures with spatially varying stiffness by changing the irradiation parameters during the process, without the need for multiple fabrications with different materials ${ }^{42}$.

In addition, we provide here the dynamic characterization of the material in the high-frequency domain (up to several hundred kilohertz), which is the natural range where microstructures resonate mechanically. These results will prove useful for the development and fabrication by $2 \mathrm{PP}$ of custom, fully-3D, and cost-effective plastic micro-electro-mechanical systems (MEMS) currently investigated at larger scales ${ }^{43-46}$.

\section{ACKNOWLEDGMENTS}

Stimulating discussions with Prof. Alberto Corigliano and Dr. Tommaso Zandrini are gratefully acknowledged. The Authors also thank Claudio Conci from Politecnico di Milano for providing the SZ2080 photoresist.

\section{DATA AVAILABILITY}

The data that support the findings of this study are available from the corresponding author upon reasonable request.

\section{REFERENCES}

${ }^{1}$ L. Jonušauskas, D. Gailevičius, S. Rekštyte, T. Baldacchini, S. Juodkazis, and M. Malinauskas, "Mesoscale laser 3d printing," Opt. Express 27, 15205-15221 (2019)

${ }^{2}$ M. Malinauskas, M. Farsari, A. Piskarskas, and S. Juodkazis, "Ultrafast laser nanostructuring of photopolymers: A decade of advances," Physics Reports 533, 1-31 (2013)

${ }^{3}$ S. Maruo and S. Kawata, "Two-photon absorbed photopolymerization for three-dimensional microfabrication," in Proc. IEEE Int. Workshop on Micro Electro Mechanical Syst. , 169-174 (1997).

${ }^{4}$ M. Farsari, M. Vamvakaki, and B. N. Chichkov, "Multiphoton polymerization of hybrid materials," Journal of Optics 12 (2010).

${ }^{5}$ T. Baldacchini, Three-Dimensional Microfabrication Using Two-Photon Polymerization, edited by A. . Elsevier (2015).

${ }^{6}$ D. Perevoznik, R. Nazir, R. Kiyan, K. Kurselis, B. Koszarna, D. T. Gryko, and B. N. Chichkov, "High-speed two-photon polymerization 3d printing with a microchip laser at its fundamental wavelength," Opt. Express 27, 25119-25125 (2019).

${ }^{7}$ A. Ovsianikov, B. Chichkov, O. Adunka, H. Pillsbury, A. Doraiswamy, and R. J. Narayan, "Rapid prototyping of ossicular replacement prostheses," Applied Surface Science 253, 6603-6607 (2007).

${ }^{8}$ A. Ovsianikov, B. Chichkov, P. Mente, N. A. Monteiro-Riviere, A. Doraiswamy, and R. J. Narayan, "Two photon polymerization of polymerceramic hybrid materials for transdermal drug delivery," International Journal of Applied Ceramic Technology 4, 22-29 (2007).

${ }^{9}$ M. M. Nava, N. Di Maggio, T. Zandrini, G. Cerullo, R. Osellame, I. Martin, and M. T. Raimondi, "Synthetic niche substrates engineered via twophoton laser polymerization for the expansion of human mesenchymal stromal cells," Journal of Tissue Engineering and Regenerative Medicine 11 2836-2845 (2017)

${ }^{10}$ M. T. Raimondi, S. M. Eaton, M. M. Nava, M. Laganà, G. Cerullo, and R. Osellame, "Two-photon laser polymerization: from fundamentals to biomedical application in tissue engineering and regenerative medicine," Journal of Applied Biomaterials \& Biomechanics , 0-0 (2012).

${ }^{11}$ M. Malinauskas, P. Danilevičius, D. Baltriukiene, M. Rutkauskas, A. Žukauskas, Z. Kairyte, G. Bičkauskaite, V. Purlys, D. Paipulas, V. Bukelskiene, and R. Gadonas, "3D artificial polymeric scaffolds for stem cell growth fabricated by femtosecond laser," Lithuanian Journal of Physics 50, 75-82 (2010)

${ }^{12}$ G. Flamourakis, I. Spanos, Z. Vangelatos, P. Manganas, L. Papadimitriou, C. Grigoropoulos, A. Ranella, and M. Farsari, "Laser-made 3d auxetic metamaterial scaffolds for tissue engineering applications," Macromolecular Materials and Engineering 305, 2000238 (2020). 
${ }^{13}$ A. S. Cordeiro, I. A. Tekko, M. H. Jomaa, L. Vora, E. McAlister, F. VolpeZanutto, M. Nethery, P. T. Baine, N. Mitchell, D. W. McNeill, and R. F. Donnelly, "Two-photon polymerisation 3d printing of microneedle array templates with versatile designs: Application in the development of polymeric drug delivery systems," Pharmaceutical Research 37, 174 (2020).

${ }^{14}$ R. A. Rezende, F. D. Pereira, V. Kasyanov, A. Ovsianikov, J. Torgensen, P. Gruber, J. Stampfl, K. Brakke, J. A. Nogueira, V. Mironov, and J. V. da Silva, "Design, physical prototyping and initial characterisation of 'lockyballs,', Virtual and Physical Prototyping 7, 287-301 (2012).

${ }^{15}$ J. Mačiulaitis, M. Deveikytė, S. Rekštytė, M. Bratchikov, A. Darinskas, A. Šimbelyte, G. Daunoras, A. Laurinavičienè, A. Laurinavičius, R. Gudas, M. Malinauskas, and R. Mačiulaitis, "Preclinical study of SZ2080 material $3 \mathrm{~d}$ microstructured scaffolds for cartilage tissue engineering made by femtosecond direct laser writing lithography," Biofabrication 7, 015015 (2015).

${ }^{16}$ M. P. Joshi, H. E. Pudavar, J. Swiatkiewicz, P. N. Prasad, and B. A. Reianhardt, "Three-dimensional optical circuitry using two-photon-assisted polymerization," Applied Physics Letters 74, 170-172 (1999).

${ }^{17}$ S. Klein, A. Barsella, H. Leblond, H. Bulou, A. Fort, C. Andraud, G. Lemercier, J. C. Mulatier, and K. Dorkenoo, "One-step waveguide and optical circuit writing in photopolymerizable materials processed by twophoton absorption," Applied Physics Letters 86, 1-3 (2005).

${ }^{18}$ M. Malinauskas, H. Gilbergs, A. Ukauskas, V. Purlys, D. Paipulas, and R. Gadonas, "A femtosecond laser-induced two-photon photopolymerization technique for structuring microlenses," Journal of Optics 12 (2010).

${ }^{19}$ L. Amato, Y. Gu, N. Bellini, S. M. Eaton, G. Cerullo, and R. Osellame, "Integrated three-dimensional filter separates nanoscale from microscale elements in a microfluidic chip," Lab on a Chip 12, 1135-1142 (2012).

${ }^{20}$ Y. J. Liu, J. Y. Yang, Y. M. Nie, C. H. Lu, E. D. Huang, C. S. Shin, P. Baldeck, and C. L. Lin, "A simple and direct reading flow meter fabricated by two-photon polymerization for microfluidic channel," Microfluidics and Nanofluidics 18, 427-431 (2015).

${ }^{21}$ F. Perrucci, V. Bertana, S. Marasso, G. Scordo, S. Ferrero, C. Pirri, M. Cocuzza, A. El-Tamer, U. Hinze, B. Chichkov, G. Canavese, and L. Scaltrito, "Optimization of a suspended two photon polymerized microfluidic filtration system," Microelectronic Engineering 195, 95 - 100 (2018).

${ }^{22}$ T. Ikegami, R. Ozawa, M. P. Stocker, K. Monaco, J. T. Fourkas, and S. Maruo, "Development of optically-driven metallic microrotors using two-photon microfabrication," Journal of Laser Micro Nanoengineering 8 , 6-10 (2013).

${ }^{23}$ S. Maruo, K. Ikuta, and H. Korogi, "Submicron manipulation tools driven by light in a liquid," Applied Physics Letters 82, 133-135 (2003).

${ }^{24}$ R. Houbertz, P. Declerck, S. Passinger, A. Ovsianikov, J. Serbin, and B. N. Chichkov, "Investigations on the generation of photonic crystals using twophoton polymerization (2PP) of inorganic-organic hybrid polymers with ultra-short laser pulses," Physica Status Solidi (A) Applications and Materials Science 204, 3662-3675 (2007).

${ }^{25}$ H. B. Sun, S. Matsuo, and H. Misawa, "Three-dimensional photonic crystal structures achieved with two-photon-absorption photopolymerization of resin," Applied Physics Letters 74, 786-788 (1999).

${ }^{26} \mathrm{C}$. LaFratta and T. Baldacchini, "Two-photon polymerization metrology: Characterization methods of mechanisms and microstructures," Micromachines 8, 101 (2017).

${ }^{27}$ E. D. Lemma, F. Rizzi, T. Dattoma, B. Spagnolo, L. Sileo, A. Qualtieri, M. D. Vittorio, and F. Pisanello, "Mechanical Properties Tunability of Three-Dimensional Polymeric Structures in Two-Photon Lithography," IEEE Trans. NANOTECHNOLOGY, 16, 23-31 (2017).

${ }^{28}$ L. J. Jiang, Y. S. Zhou, W. Xiong, Y. Gao, X. Huang, L. Jiang, T. Baldacchini, J.-F. Silvain, and Y. F. Lu, "Two-photon polymerization: investigation of chemical and mechanical properties of resins using raman mi- crospectroscopy," Opt. Lett. 39, 3034-3037 (2014).

${ }^{29}$ K. Cicha, T. Koch, J. Torgersen, Z. Li, R. Liska, and J. Stampfl, "Young ' s modulus measurement of two- photon polymerized micro-cantilevers by using nanoindentation equipment,” J. Appl. Phys. 112, 094906 (2012).

${ }^{30}$ J. Bauer, A. G. Izard, Y. Zhang, T. Baldacchini, and L. Valdevit, "Programmable Mechanical Properties of Two-Photon Polymerized Materials : From Nanowires to Bulk,” Adv. Mater. Technol. 1900146, 1-11 (2019).

${ }^{31}$ M. Farsari and B. N. Chichkov, "Two-photon fabrication," Nature Photonics 3, 450-452 (2009)

${ }^{32}$ A. Skarmoutsou, G. Lolas, C. A. Charitidis, M. Chatzinikolaidou, M. Vamvakaki, and M. Farsari, "Nanomechanical properties of hybrid coatings for bone tissue engineering," J. Mech. Behav. Biomed. Mater. 25, 48-62 (2013).

${ }^{33}$ T. Tickunas, M. Perrenoud, S. Butkus, R. Gonadas, D. Paipulas, Y. Bellouard, and V. Sirutkaitis, "Combination of additive and subtractive laser 3D microprocessing in hybrid glass / polymer microsystems for chemical sensing applications," Opt. Express 25, 26280-26288 (2017)

${ }^{34}$ M. Carlotti and V. Mattoli, "Functional materials for two-photon polymerization in microfabrication," Small 15, 1902687 (2019).

${ }^{35}$ M. Tromayer, P. Gruber, A. Rosspeintner, A. Ajami, W. Husinsky, F. Plasser, L. González, E. Vauthey, A. Ovsianikov, and R. Liska, "Wavelength-optimized two-photon polymerization using initiators based on multipolar aminostyryl-1,3,5-triazines," Scientific Reports 8, 17273 (2018).

${ }^{36}$ A. Ovsianikov, J. Viertl, B. Chichkov, M. Oubaha, B. MacCraith, I. Sakellari, A. Giakoumaki, D. Gray, M. Vamvakaki, M. Farsari, and C. Fotakis, "Ultra-low shrinkage hybrid photosensitive material for two-photon polymerization microfabrication," ACS Nano 2, 2257-2262 (2008).

${ }^{37}$ M. T. Raimondi, M. M. Nava, S. M. Eaton, A. Bernasconi, K. C. Vishnubhatla, G. Cerullo, and R. Osellame, "Optimization of femtosecond laser polymerized structural niches to control mesenchymal stromal cell fate in culture," Micromachines 5, 341-358 (2014).

${ }^{38}$ L. J. Jiang, J. H. Campbell, Y. F. Lu, T. Bernat, and N. Petta, "Direct writing target structures by two-photon polymerization," Fusion Science and Technology 70, 295-309 (2016).

${ }^{39}$ S. Chung and S. Park, "Effects of temperature on mechanical properties of SU-8 photoresist material," J. Mech. Sci. Technol. , 2701-2707 (2013).

${ }^{40}$ A. Caspani, C. Comi, A. Corigliano, G. Langfelder, V. Zega, and S. Zerbini, "Dynamic nonlinear behavior of torsional resonators in MEMS," J. Micromechanics Microengineering 24 (2014).

${ }^{41}$ Z. Bayindir, Y. Sun, M. J. Naughton, C. N. LaFratta, T. Baldacchini, J. T. Fourkas, J. Stewart, B. E. A. Saleh, and M. C. Teich, "Polymer microcantilevers fabricated via multiphoton absorption polymerization," Applied Physics Letters 86, 064105 (2005).

${ }^{42}$ F. Klein, B. Richter, T. Striebel, C. M. Franz, G. v. Freymann, M. Wegener, and M. Bastmeyer, "Two-component polymer scaffolds for controlled three-dimensional cell culture," Advanced Materials 23, 1341-1345 (2011).

${ }^{43}$ P. Pan, J. Qu, W. Zhang, X. Dong, W. Wei, C. Ru, and X. Liu, "Robotic stimulation of freely moving drosophila larvae using a 3d-printed micro force sensor," IEEE Sensors Journal 19, 3165-3173 (2019).

${ }^{44}$ V. Zega, M. Invernizzi, R. Bernasconi, F. Cuneo, G. Langfelder, L. Magagnin, M. Levi, and A. Corigliano, "The first 3d-printed and wet-metallized three-axis accelerometer with differential capacitive sensing," IEEE Sensors J. 19, 9131-9138 (2019).

${ }^{45}$ L. Faller, M. Lenzhofer, C. Hirschl, M. Kraft, and H. Zangl, "Characterization of a robust 3d- and inkjet-printed capacitive position sensor for a spectrometer application," Sensors (Basel) 22, 443 (2019).

${ }^{46}$ L. Gaffuri Pagani, P. Carulli, V. Zega, R. Suriano, R. Bernasconi, A. Frangi, M. Levi, L. Magagnin, and G. Langfelder, "The first three-dimensional printed and wet-metallized coriolis mass flowmeter," IEEE Sensors Lett. 4 2500604 (2020). 


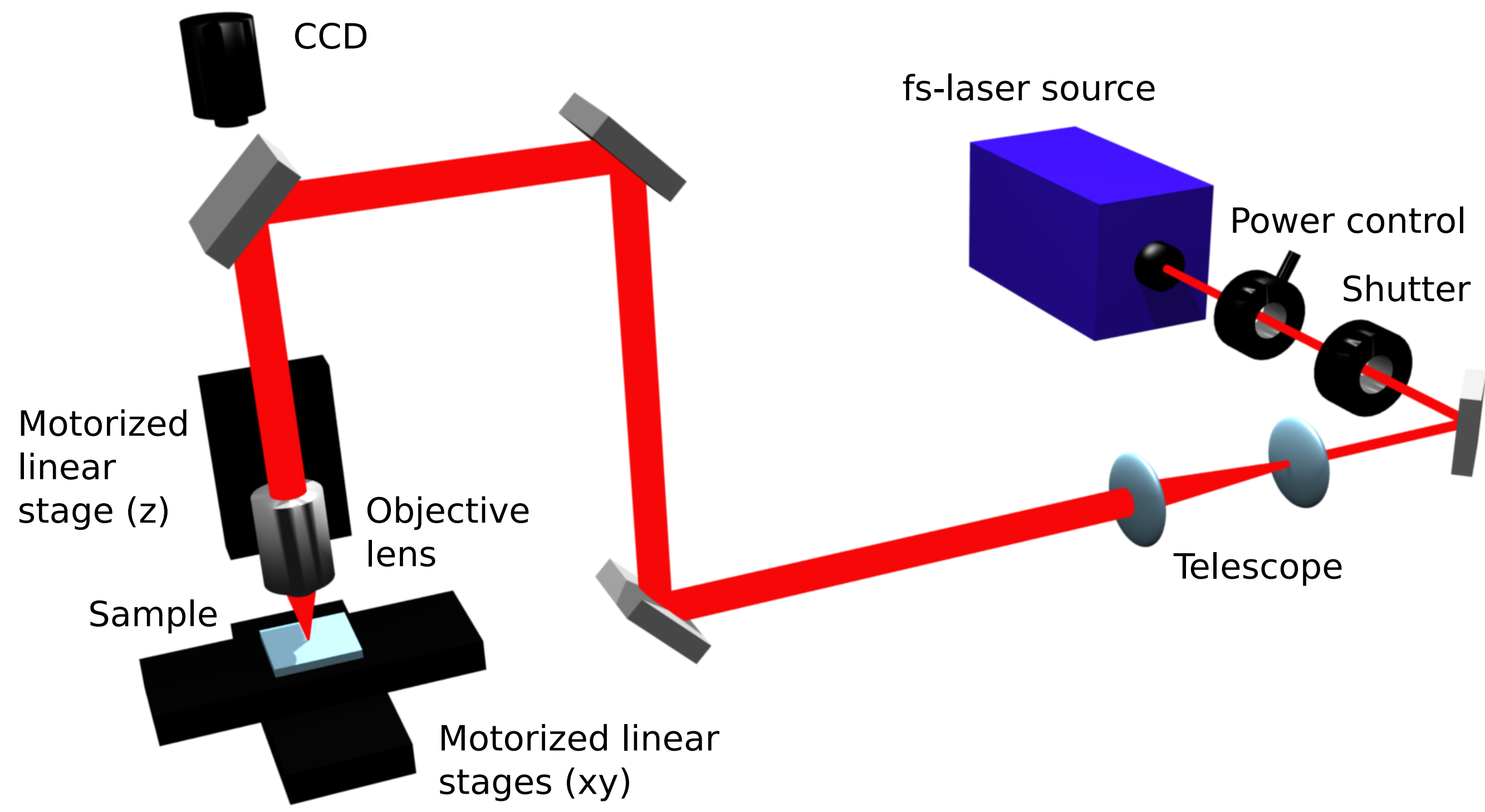




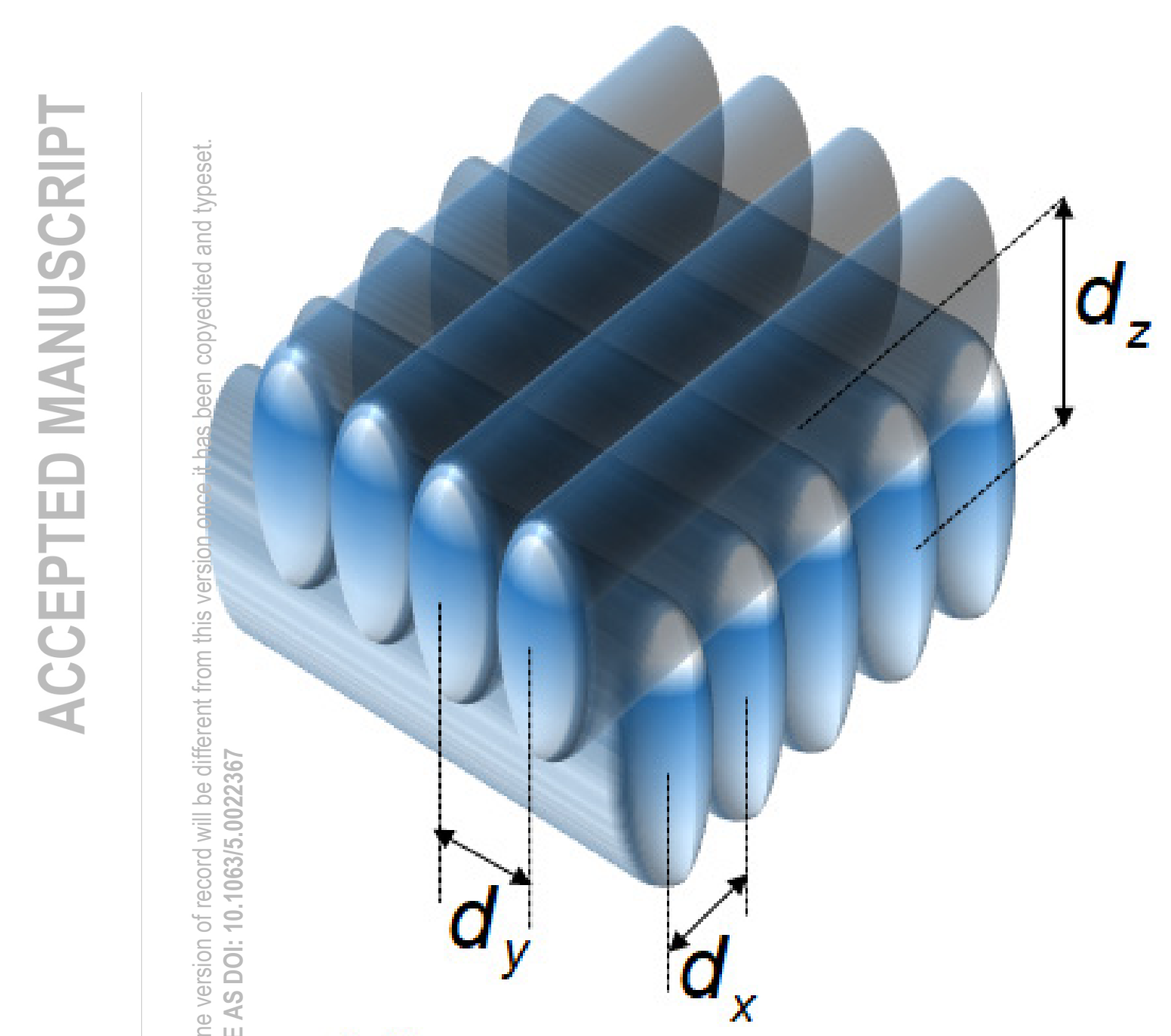

(a)

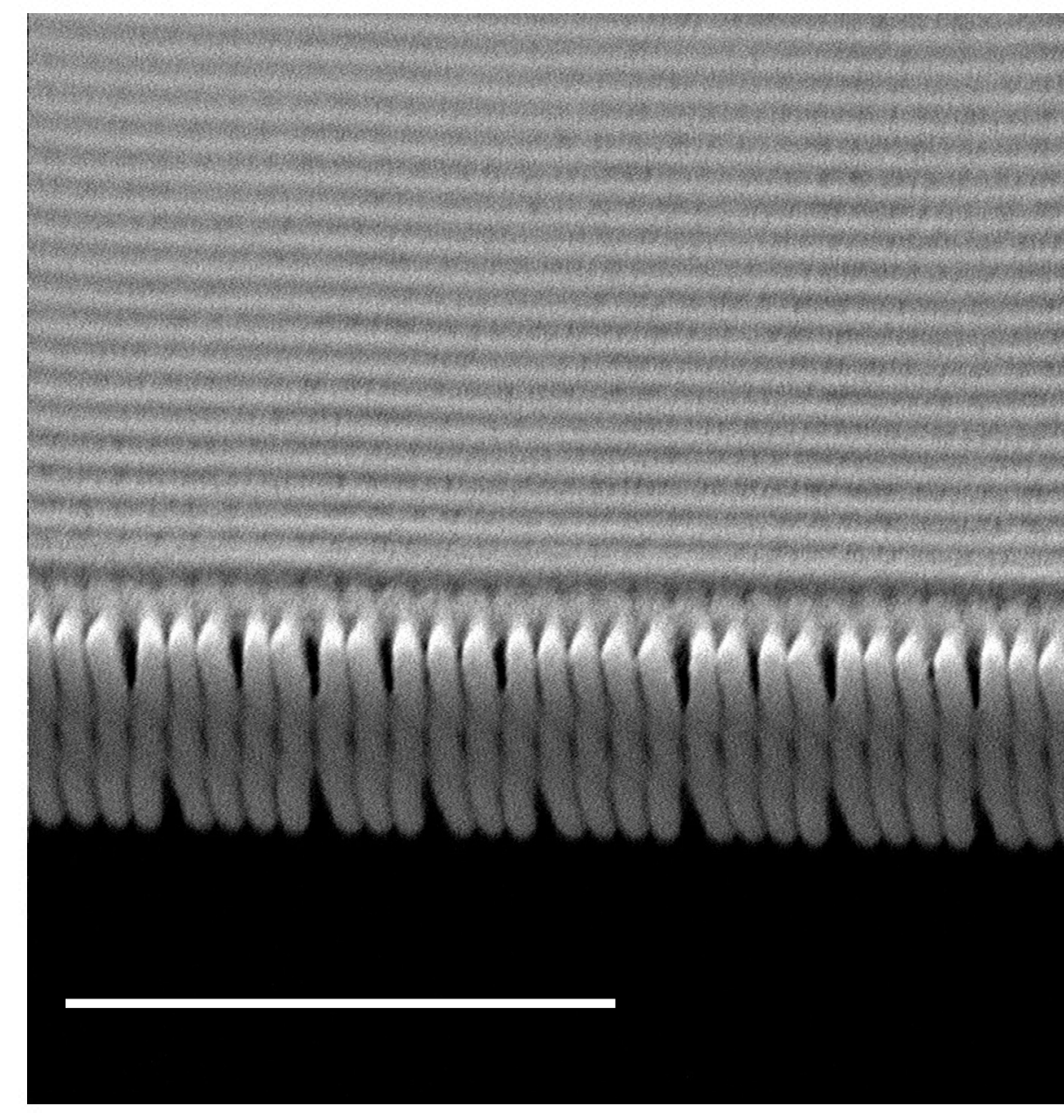

(b)

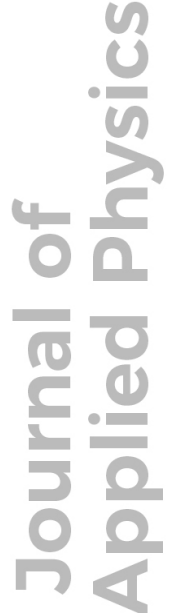

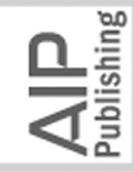




\section{photodiode}

- iris
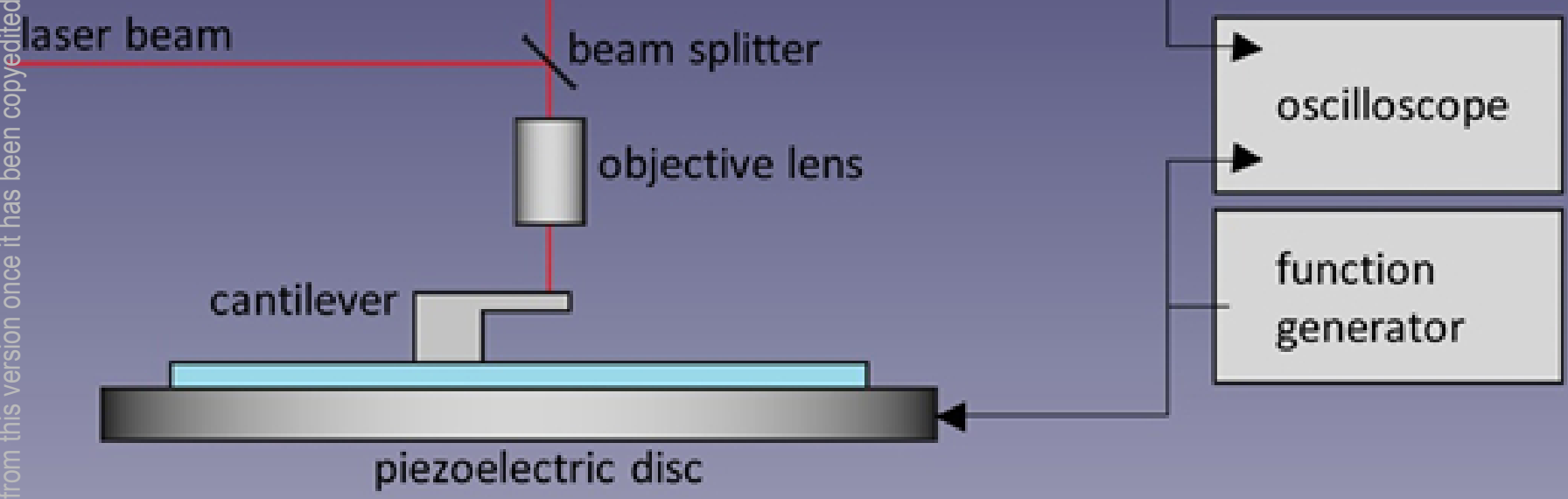

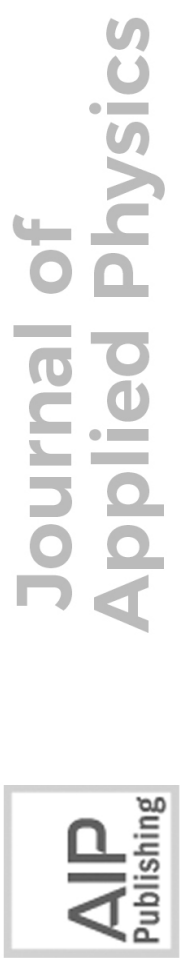


(a)

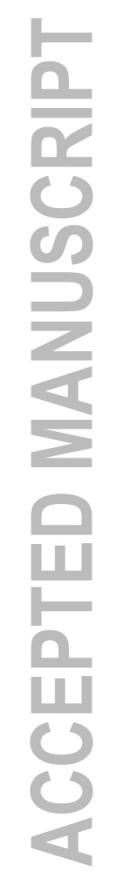

$$
\text { (a) }
$$

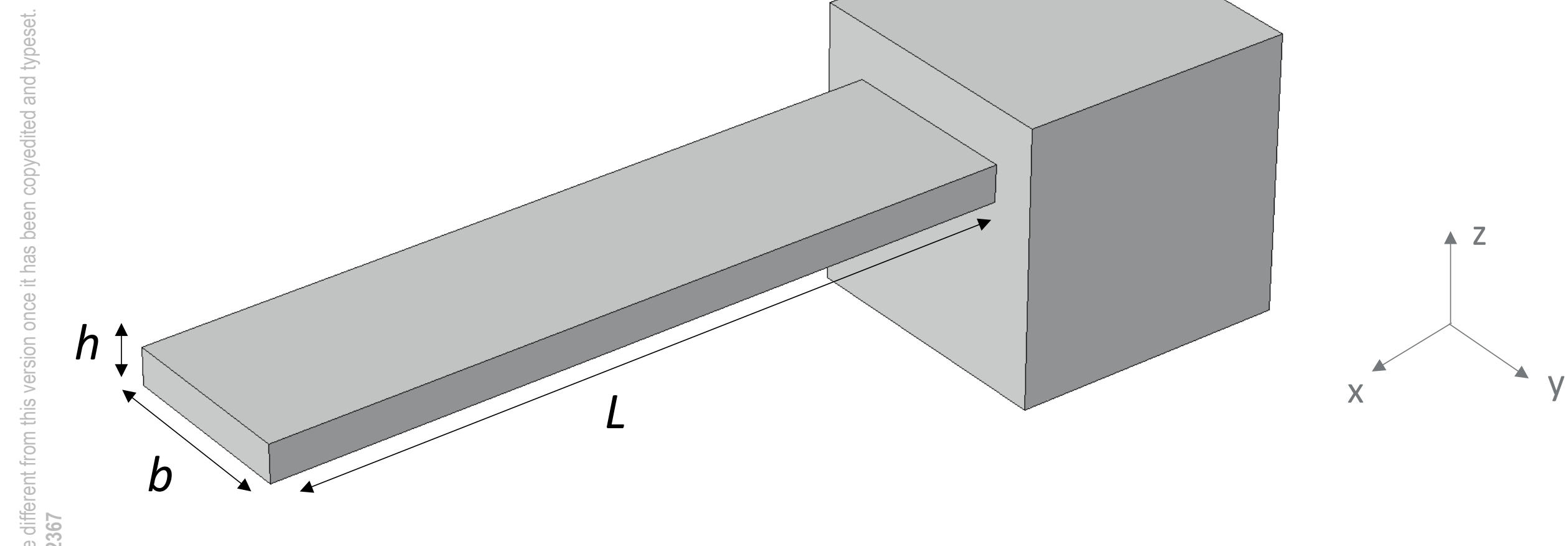

(b)

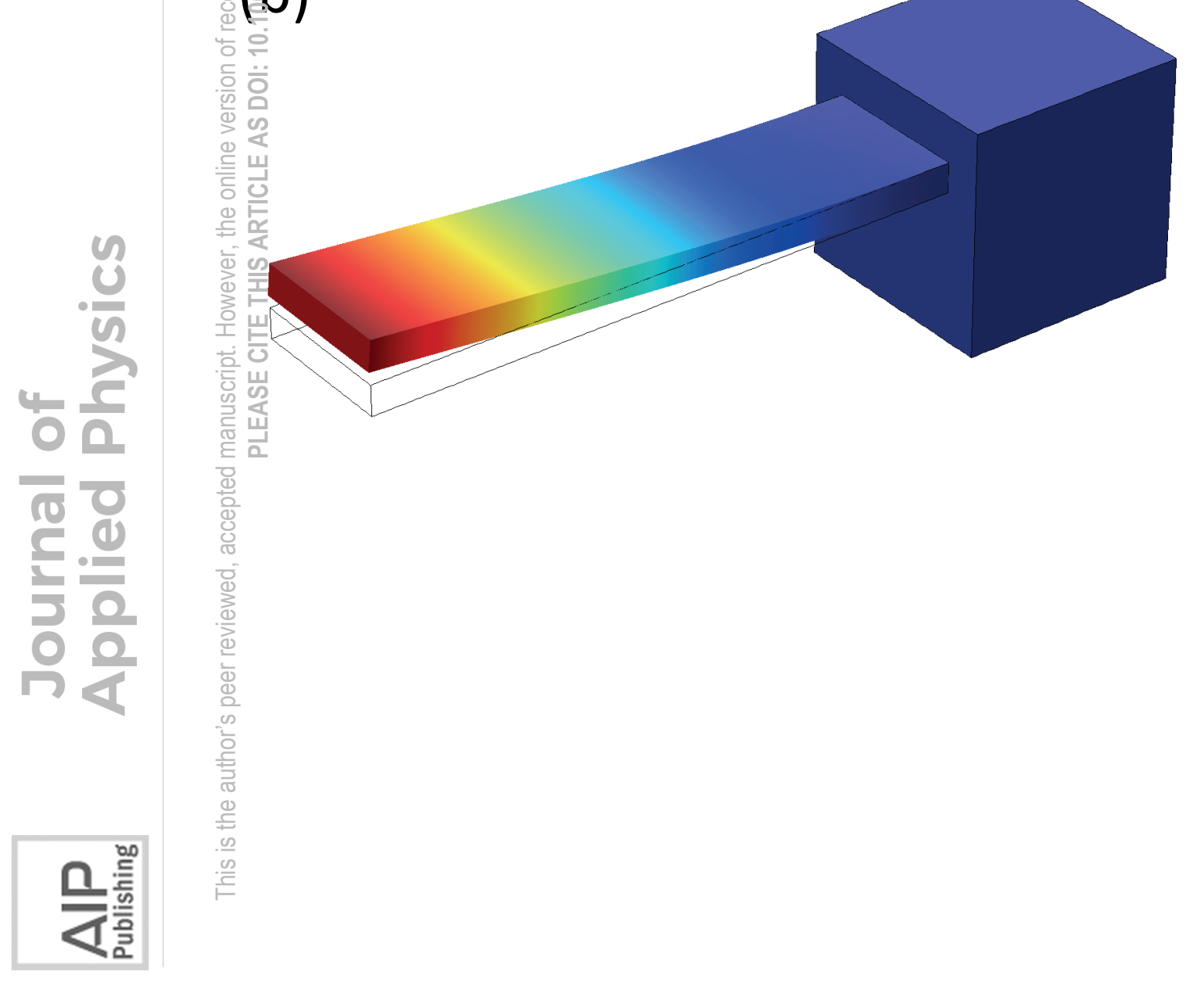

(c) 
(a)

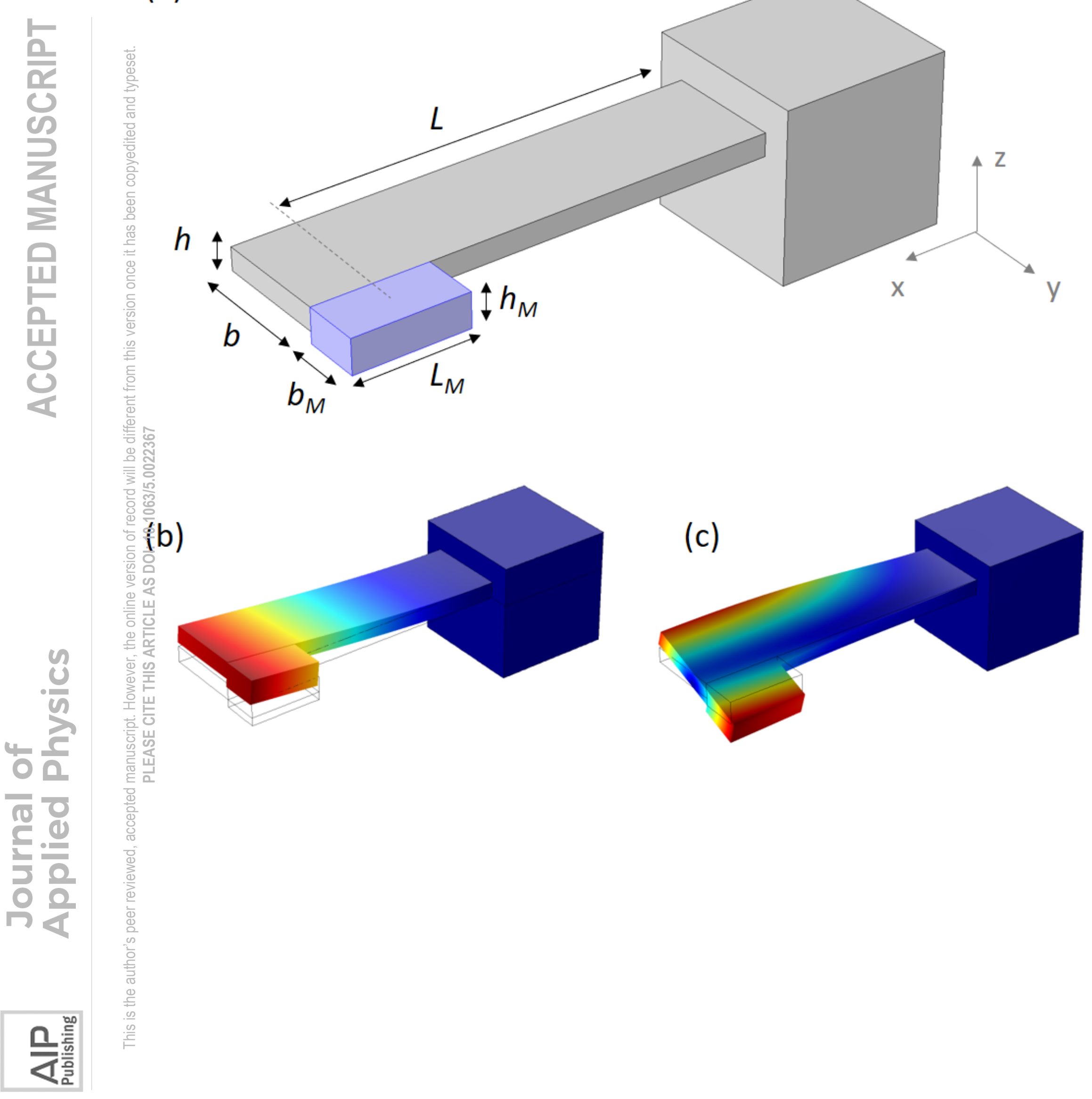


(a)
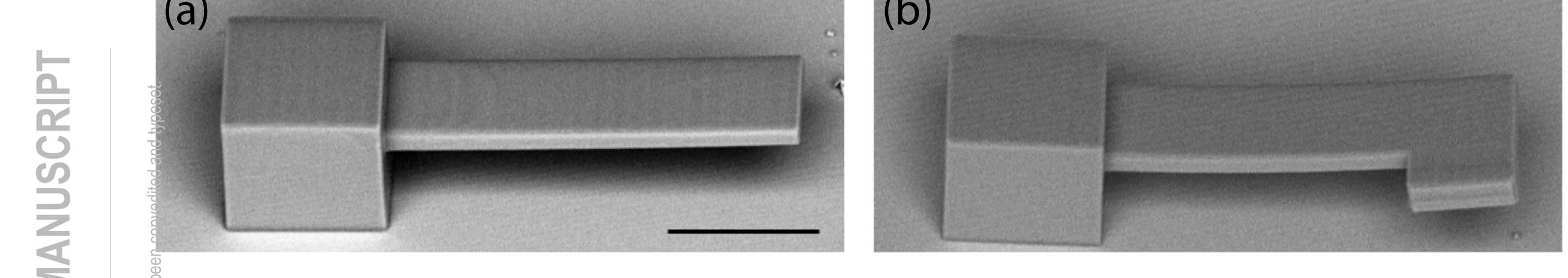

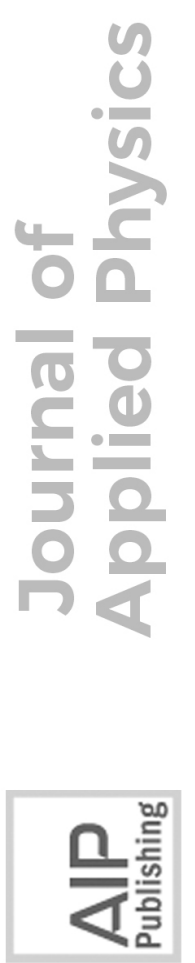




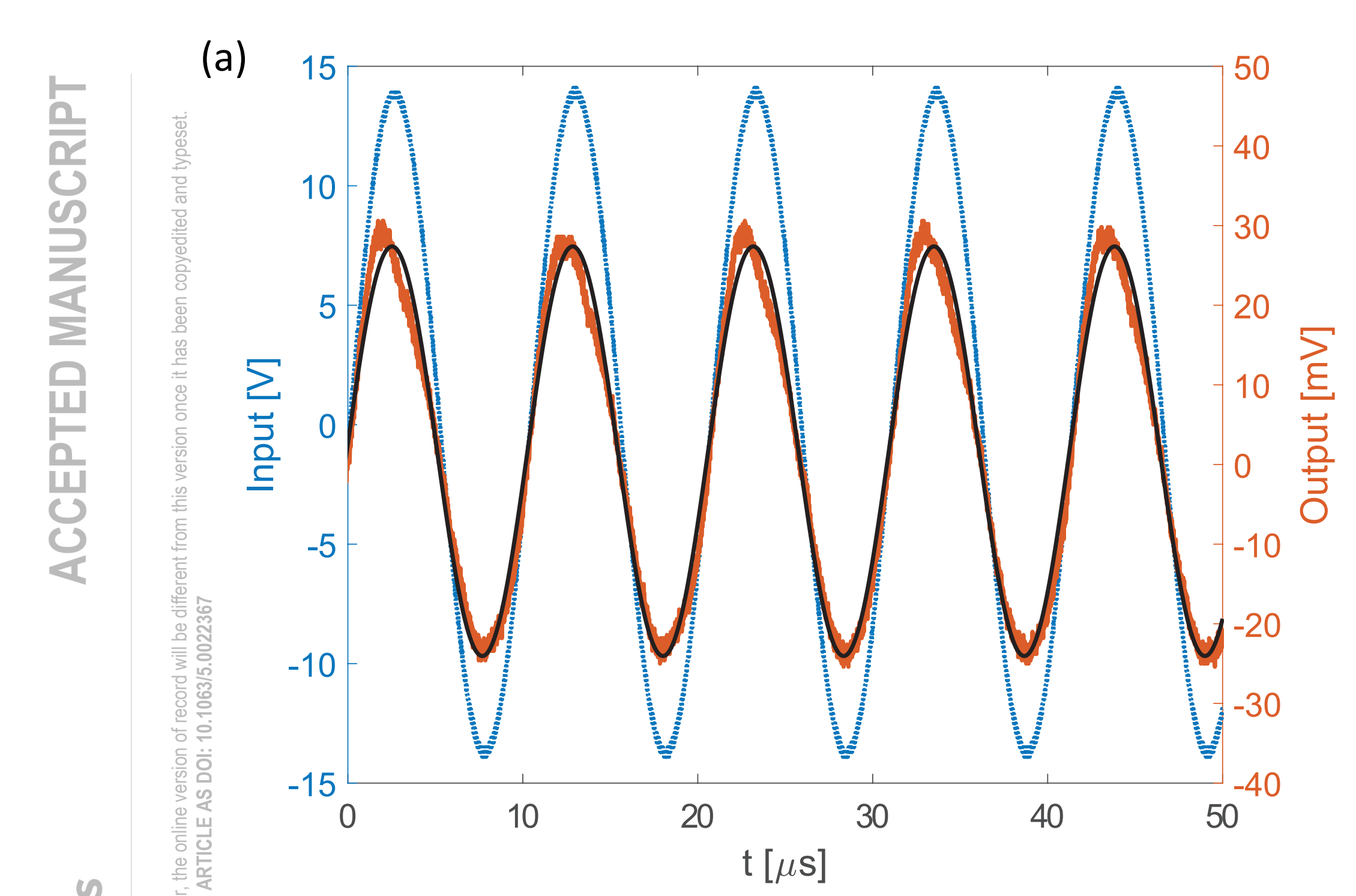

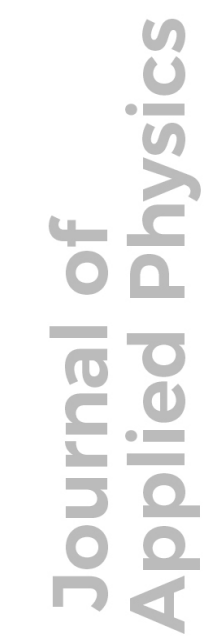

(b)

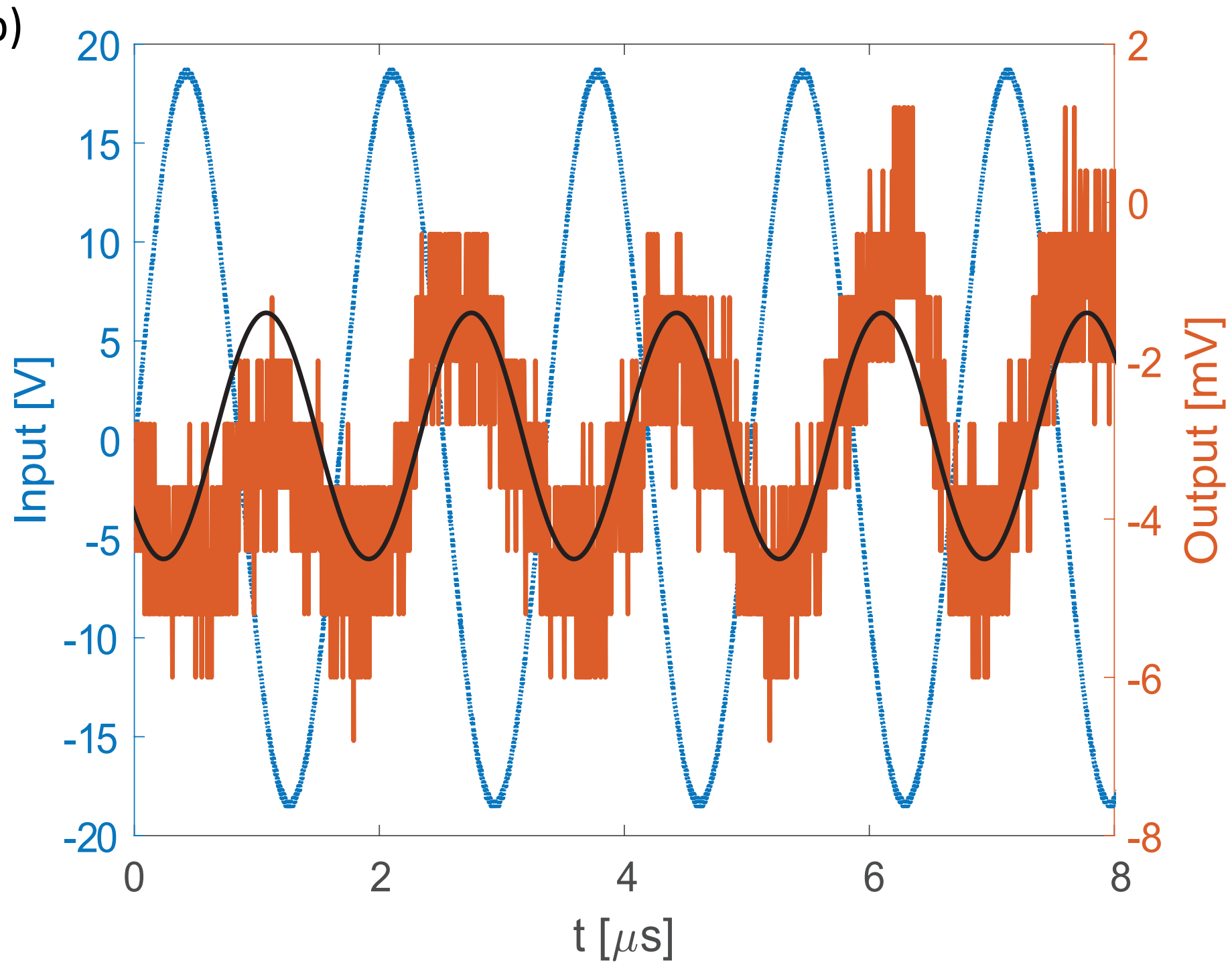




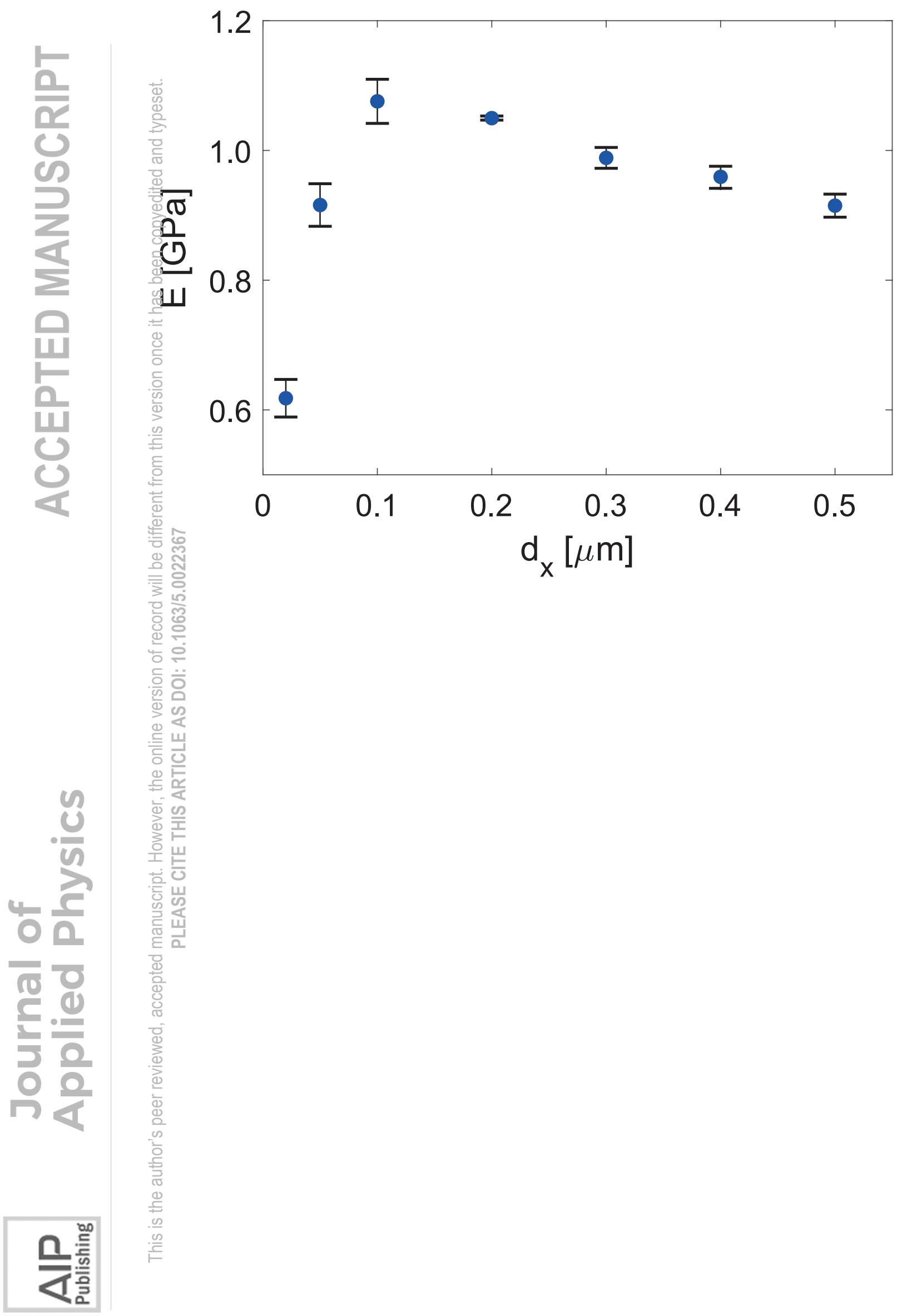




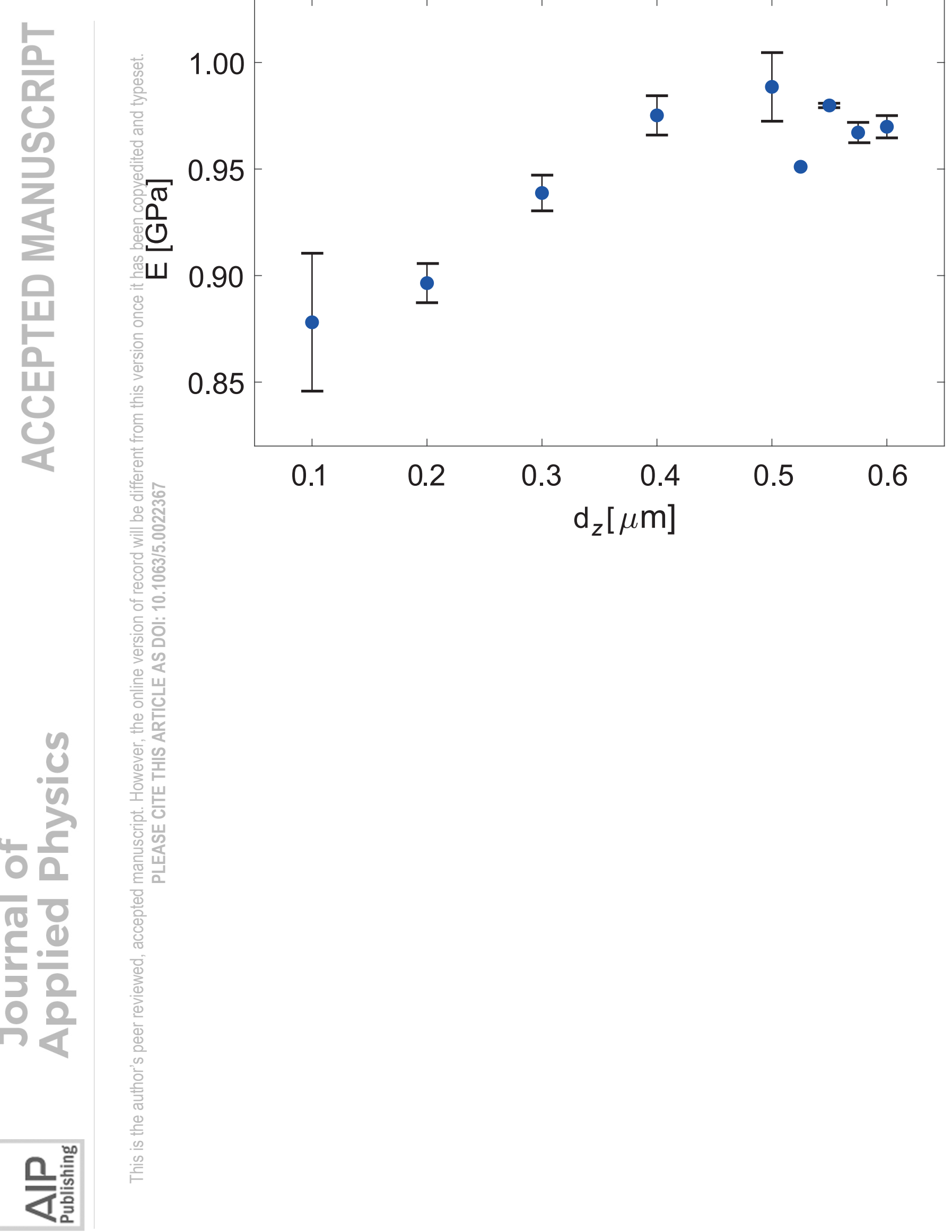




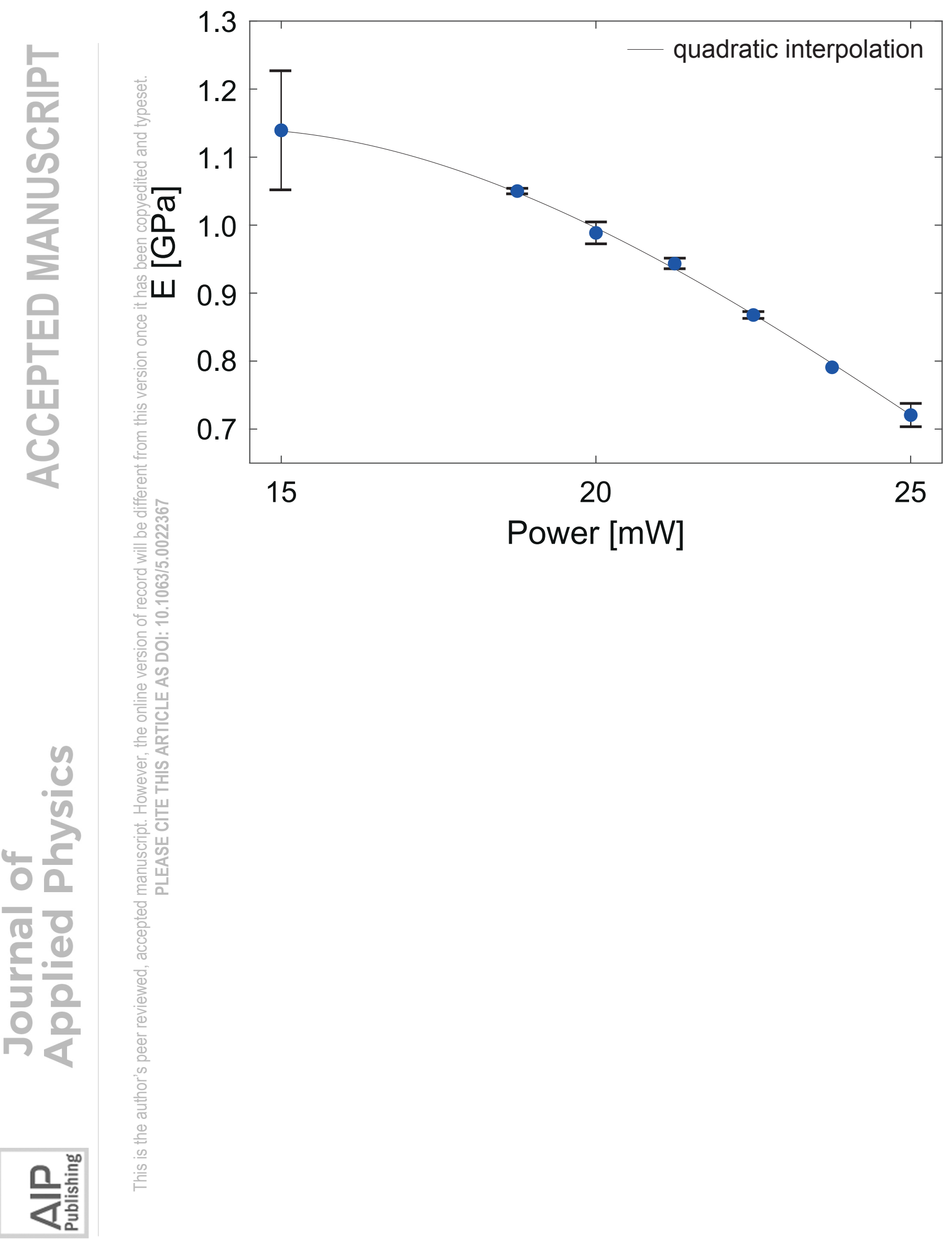




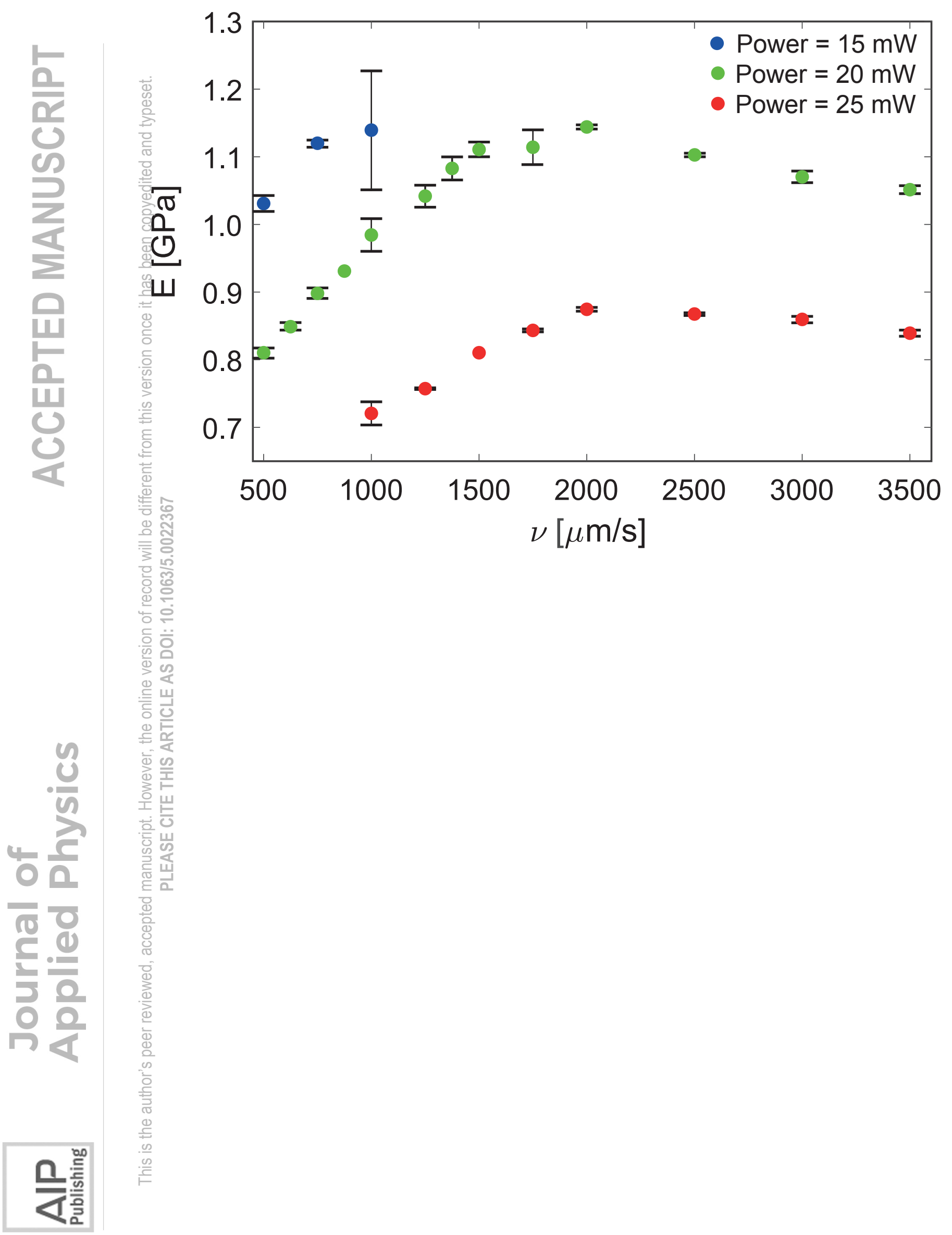

